\title{
Universal relation between the density and the viscosity of dispersions of nanoparticles and stabilized emulsions
}

\author{
Hatim Machrafi ${ }^{1,2, *}$
}

\author{
${ }^{1}$ Thermodynamique des Phénomènes Irréversibles, Institut de Physique, Université de Liège, \\ Liège 4000, Belgium \\ ${ }^{2}$ Service Chimie-Physique, Ecole Polytechnique, Université libre de Bruxelles, Bruxelles \\ 1050, Belgium \\ ${ }^{3}$ GIGA-In Silico Medicine, Université de Liège, Liège 4000, Belgium \\ *Corresponding author. E-mail: h.machrafi@uliege.be
}

\begin{abstract}
The effective viscosity of nanoparticle dispersions has been investigated experimentally quite a lot and various behaviours have been observed. Many models have been proposed to predict the effective viscosity, but these are mainly empirical ones, correlations with a tuning parameter or based on fastidious molecular interactions simulations. In this work, we propose a new fully physics-based analytical expression for the effective viscosity implementing theories from extended thermodynamics, including nano-confinement effects, nanoparticle-fluid interactions, density effects, size effects and nanoparticle volume fraction. We validate this model against several different types of nanoparticle dispersions and emulsions and explain the different behaviours using the same model. It appears that the density ratio of the nanoparticles with respect to the fluid plays a crucial role affecting the viscosity. The nanoparticle-fluid interactions become increasingly important for smaller nanoparticle sizes. From these comparisons, we arrive at a universal simplified expression for the effective viscosity of nanoparticle dispersions, where it is observed that there is a direct universal relation between the nanoparticles and fluid densities and the nanodispersion viscosities. The validity of such a relation has been explicitly demonstrated.
\end{abstract}

Keywords: Effective viscosity, Nanodispersions, Extended thermodynamics, Nano and micro emulsions, Density effect

\section{Introduction}

Viscosity is a central property in the investigation of mechanical and thermal properties of nanoparticle dispersions. These nanodispersions, when concerning dispersions of solid particles in a liquid, are also called nanofluids. For many applications, nanodispersions are used to enhance heat transfer for solar energy harvesting and storage, provide for smart cooling, or control the thermal management for electronics [1-6]. Nanoparticle dispersions are also used as a precursor for the preparation of nanocomposites or self-assembled structures in view of other applications, e.g. supercapacitors, batteries, electrodes or membranes [7-9]. Liquid-liquid nanodispersions find particular applications in the medical sector, as means for drug delivery or pharmaceutics, cosmetics and food [10-12]. The required effects depend heavily on the presence of the nanoparticles in the fluid, so that it is important that those nanoparticles are well dispersed. For that, they need to be well stabilized in order to avoid flocculation, settlement or agglomeration, which is quite a challenge [13]. Some dispersion mechanisms exist, such as electrostatic, steric, or electro-steric stabilization. These mechanisms rely on inter-particle repulsive forces (nanoparticle surface treatment by e.g. adsorption of ions, physical adsorption 
of charged species, accumulation or depletion of electrons), van der Waals inter-particle screening by macromolecular barriers attached to the nanoparticle surfaces and a combination of both, where surfactants or dispersing agents can also play a role [2]. These stabilization mechanisms suggest that the nanofluids are not really entropically mixed but rather dispersed. This latter observation is of great importance for the thermophysical properties of nanodispersions, which should be well defined or at least known for the respective applications. Particular interest is given here fore the effective viscosity. Many experiments have been performed in order to characterize the effective viscosity $\mu_{n f}$ as a function of the amount of nanoparticles, often expressed in the volume fraction. Although the vast majority of the data show an increasing tendency, the viscosity increments differ considerably from one nanodispersion to another [14-20]. Fig. 1 shows some examples of the relative effective viscosity $\mu_{n f} / \mu_{f}$ for some nanodispersions, where $\mu_{f}$ is the viscosity of the base fluid.

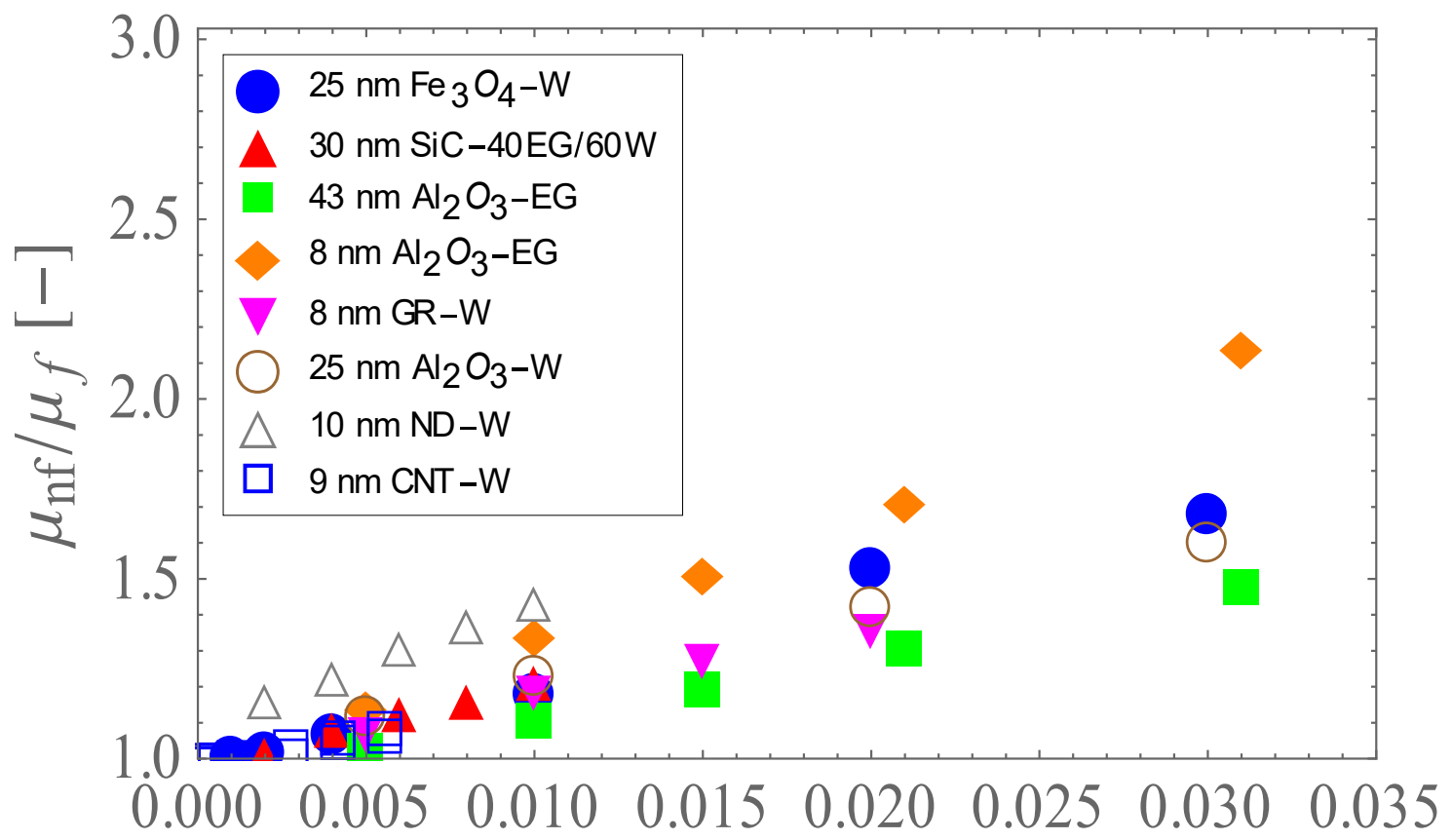

$\varphi$

Figure 1: Experimental relative effective viscosity as a function of the volume fraction of nanoparticle dispersions. The legend shows the data in the form "size of nanoparticles" "type of nanoparticles" "type of fluid", where W stands for water and EG for ethylene glycol with one W/EG mixture given in volumetric contents. The nanodispersions are: $25 \mathrm{~nm} \mathrm{Fe} \mathrm{O}_{4}-\mathrm{W}$ [14], $30 \mathrm{~nm} \mathrm{SiC}-40 \mathrm{EG} / 60 \mathrm{~W}$ [15], $43 \mathrm{~nm} \mathrm{Al}_{2} \mathrm{O}_{3}$-EG [16], $8 \mathrm{~nm} \mathrm{Al}_{2} \mathrm{O}_{3}$-EG [16], $8 \mathrm{~nm}$ GR-W [17], $25 \mathrm{~nm} \mathrm{Al}_{2} \mathrm{O}_{3}-\mathrm{W}$ [18], $10 \mathrm{~nm} \mathrm{ND-W} \mathrm{[19],} 9 \mathrm{~nm}$ CNT-W [20], where GR stands for graphite, ND for nanodiamonds and CNT for carbon nanotubes.

From Fig. 1, we can see that comparing, for instance, the nanodispersions $25 \mathrm{~nm} \mathrm{Fe} 3 \mathrm{O}_{4}-\mathrm{W}$ and $25 \mathrm{~nm} \mathrm{Al}_{2} \mathrm{O}_{3}-\mathrm{W}$ the former has a higher effective viscosity than the latter. The only difference being the type of nanoparticle, the difference could lie in the density of the nanoparticle and/or their interaction with the base fluid. From the comparison between the nanodispersions $43 \mathrm{~nm}$ $\mathrm{Al}_{2} \mathrm{O}_{3}$-EG and $8 \mathrm{~nm} \mathrm{Al}_{2} \mathrm{O}_{3}$-EG, we can also see that the nanoparticle size can play a role. $\mathrm{A}$ useful tool to understand and predict the mechanisms that result into the behavior of the effective viscosity of nanodispersions is to propose a model, based on physical and fundamental principles. The latter aspect causes such a model to be universally applicable for different types 
of nanosdispersions. Furthermore, the model should be as less complicated as possible, which would make it more easily applicable in numerical 2D or 3D models or for industrial use. The aim can then be resumed to propose a physics-based analytical expression for the effective viscosity of nanodispersions. Most studies are based on Einstein's model [21], which has served as inspiration for most descriptions used today. The subject is of interest as confirmed by the numerous papers published during the last decade, e.g.[22-28]. However, many of them rest on ad hoc correlations with the shortcoming of being of limited applicability and generally lack of physical background. In that respect, the present approach is different and original as it is grounded on a modern non-equilibrium thermodynamic theory, more specifically Extended Non-Equilibrium/Irreversible Thermodynamics [29,30], the main idea underlying this formalism is to elevate the dissipative fluxes, like the heat and matter fluxes and the viscous stress tensor to the status of independent variables. As such, we propose a physics-based approach, without adjustment parameters or empirical correlations, and lay out the effect of other material properties, such as the density of the fluid and nanoparticles.

\section{Model}

\subsection{Evolution equation for the mass flux}

The nanofluid is modelled as a two-component mixture consisting of nanoparticles, with volume fraction $\varphi\left(=\frac{n_{p} V_{p}}{V_{t}}\right)$, with $n_{p}$ the number of nanoparticles, $V_{p}$ the volume of one nanoparticle and $V_{t}$ the total volume $V_{t}=n_{p} V_{p}+V_{f}$, where $V_{f}$ is the fluid volume), dispersed homogeneously in a Newtonian viscous incompressible fluid of mass density $\rho_{f}$ moving with a velocity $\boldsymbol{v}_{\boldsymbol{f}}$. The nanoparticles are assumed to be rigid spheres of mass density $\rho_{p}$ moving at velocity $\boldsymbol{v}_{p}$. The diffusion fluxes of the fluid and particles are defined as

$\boldsymbol{J}_{f}=(1-\varphi) \rho_{f}\left(\boldsymbol{v}_{f}-\boldsymbol{v}\right)$,

$\boldsymbol{J}_{p}=\varphi \rho_{p}\left(\boldsymbol{v}_{p}-\boldsymbol{v}\right)$

with the subscripts " $f$ " and " $p$ " denoting the base fluid and nanoparticles respectively. Bold case letters will be used throughout this work to denote vectors. The barycentric velocity $\boldsymbol{v}$ is given by

$\rho \boldsymbol{v}=(1-\varphi) \rho_{f} \boldsymbol{v}_{f}+\varphi \rho_{p} \boldsymbol{v}_{p}$

wherein the total mass density $\rho$ is $\rho=(1-\varphi) \rho_{f}+\varphi \rho_{p}$. Macroscopically, diffusion can be described by Fick's law. However, when we work with nanoparticles, we expect non-local and relaxational effects of the diffusion flux, so that we can expect that the mass flux is dissipative of nature and needs an evolution equation to be well described. Furthermore, since the mass flux is considered to be dissipative, extended non-equilibrium thermodynamics elevates it to an independent variable at the same level as the mass fraction. The entropy production takes then another form than the usual one from Gibb's equation for entropy production (see the appendix) and becomes

$\sigma^{S}=\boldsymbol{J}_{1} \cdot\left(-\frac{\nabla \eta}{T}-\rho \alpha \frac{d \boldsymbol{J}_{1}}{d t}+\gamma \nabla^{2} \boldsymbol{J}_{1}\right)+\gamma \nabla \boldsymbol{J}_{1} \otimes \nabla \boldsymbol{J}_{1} \geq 0$

where $\boldsymbol{J}_{1}$ is the dissipative mass flux of component $1, \eta=\eta_{1}-\eta_{2}$ the chemical potential difference between the two components 1 and 2 (hereafter called the chemical potential), $\alpha$ and 
$\gamma$ phenomenological coefficients to be determined, $T$ the temperature, $t$ the time and $\otimes$ standing for the tensorial product. The simplest way guaranteeing the positiveness of relation (4) is to assume that there exits a linear relation between the flux $J_{1}$ and its conjugated force represented by the terms between parenthesis and that $\gamma$ is a positive factor. To summarize, one is led to

$\boldsymbol{J}_{1}=\frac{1}{\chi}\left(-\frac{\nabla \eta}{T}-\rho \alpha \frac{d \boldsymbol{J}_{1}}{d t}+\gamma \nabla^{2} \boldsymbol{J}_{1}\right), \quad \gamma \geq 0$

with $\chi$ a positive phenomenological coefficient in order to meet the condition $\sigma^{s} \geq 0$. The term $\frac{\rho \alpha}{\chi}$ has unity time and can be defined by the mass flux relaxation time as is the tradition in the time-evolution of fluxes in EIT, so that $\frac{\rho \alpha}{\chi}=\tau$. Furthermore, it should be noted that $\nabla^{2} J_{1}$ represents the non-local contribution of the mass flux, for which we can observe that $\frac{\gamma}{\chi}$ has a unit of length to the square. Typically, in EIT, this is defined as the square of the mean free path of the mass flux. When neglecting the non-local contribution and the relaxation effect, Eq. (5) reduces to Fick's law. As such, expressing the chemical potential difference $\eta$ in terms of $c_{1}$, leading to $\nabla \eta=\left(\partial \eta / \partial c_{1}\right) \nabla c_{1}$, we can easily see that we can write

$\frac{1}{T \chi} \frac{\partial \eta}{\partial c_{1}}=\rho D$

Finally, defining the variables with subscript 1 to stand for the nanoparticles (subscript $p$ ) and the variables with subscript 2 for the fluid (subscript $f$ ), we obtain that the mass flux obeys the following time evolution equation expression

$\tau \frac{\partial \boldsymbol{J}_{p}}{\partial t}+\boldsymbol{J}_{p}=-\rho D \nabla c+\ell^{2} \nabla^{2} \boldsymbol{J}_{p}$

where we write, for simplicity, $c_{p} \equiv c$ and thus $c_{f}=1-c$. For later purposes, we find it more convenient to work with the fluid mass flux, $\boldsymbol{J}_{f}$. As $\boldsymbol{J}_{f}+\boldsymbol{J}_{p}=0$, the mass flux evolution can be written as [29-32]

$\tau \partial_{t} \boldsymbol{J}_{f}+\boldsymbol{J}_{f}=-\rho D \nabla c_{f}+\ell^{2} \nabla^{2} \boldsymbol{J}_{f}=\rho D \nabla c+\ell^{2} \nabla^{2} \boldsymbol{J}_{f}$

wherein $\tau, \partial_{t}, D, c\left(=\varphi \rho_{p} / \rho\right)$, and $\ell$ are the mass flux relaxation time, partial time derivative, diffusion coefficient, mass fraction of the fluid and the mean free path of the mass flux, respectively. Relation (8) generalizes Fick's law, which is recovered when $\tau$ and $\ell$ become negligible with respect to the characteristic time and size of the system, respectively (i.e. mathematically $\tau \partial_{t}$ and $\ell^{2} \nabla^{2}$ tend to zero). As the fluid velocity remains small, all non-linear terms in the velocity (as $\boldsymbol{v} \cdot \nabla \boldsymbol{J}$ ) are neglected, which justifies that the material time derivative is substituted by the partial time derivative. The last term in the right-hand side of (8) stands for the non-local effects, which are important in presence of nano systems.

Substituting the definition (1) of the mass flux $\boldsymbol{J}_{f}$ in (8) and assuming that the material densities and volume fractions do not change in time nor in space (closed system and perfectly mixed) leads to

$\tau(1-\varphi) \rho_{f} \partial_{t}\left(\boldsymbol{v}_{f}-\boldsymbol{v}\right)=\rho D \nabla c-(1-\varphi) \rho_{f}\left(\boldsymbol{v}_{f}-\boldsymbol{v}\right)+\ell^{2}(1-\varphi) \rho_{f} \nabla^{2}\left(\boldsymbol{v}_{f}-\boldsymbol{v}\right)$ 
We will eliminate the term in $\boldsymbol{v}$ by making use of the global momentum equation which, in absence of body forces may be written as

$\rho \partial_{t} \boldsymbol{v}=-\nabla p+\nabla \cdot \boldsymbol{\sigma}$

wherein $p$ is the pressure and $\sigma$ the stress tensor given by the following constitutive relation

$\boldsymbol{\sigma}=\mu_{f} \nabla \boldsymbol{v}_{f}+\mu_{d} \nabla\left(\boldsymbol{v}_{f}-\boldsymbol{v}_{p}\right)$

where the quantity $\mu_{f}$ is the usual fluid kinematic viscosity, while $\mu_{d}$ designates the extra viscosity resulting from the mass diffusion flux of the nanoparticles, expressed into a nanoparticle velocity, different from that of the fluid, caused by friction between the nanoparticles and the fluid. Such a contribution is new and is justified because resistance to deformation will be influenced by the presence of particles, which gives raise to strong nonlocalities expressed by the tem in $\nabla\left(\boldsymbol{v}_{f}-\boldsymbol{v}_{p}\right)$. Note that this is different from the bulk viscosity, which is zero as the fluid is assumed to be incompressible.

More information about the new coefficient $\mu_{d}$ will be given in the forthcoming. Making use of the following equivalent definition of $J_{f}$ instead of (1)

$J_{f}=\varphi(1-\varphi) \frac{\rho_{f} \rho_{p}}{\rho}\left(\boldsymbol{v}_{f}-\boldsymbol{v}_{p}\right)$

and filling relation (10) in (9), with $\sigma$ given by (11), one obtains the time evolution equation for the velocity of the base fluid, namely

$\rho \partial_{t} \boldsymbol{v}_{f}=$
$-\nabla p-\frac{\rho D}{\tau} \nabla \ln (1-c)+\mu_{f} \nabla^{2} \boldsymbol{v}_{f}-\frac{\varphi \rho_{p}}{\tau}\left(\boldsymbol{v}_{f}-\boldsymbol{v}_{p}\right)+\left(\mu_{d}+\frac{\varphi \rho_{p}}{\tau} \ell^{2}\right) \nabla^{2}\left(\boldsymbol{v}_{f}-\boldsymbol{v}_{p}\right)$

There are two unusual terms in Eq. (13), the penultimate denoting a flux term and the last a diffusional flux term. The flux term stands for the kinetic force related to the partice-fluid velocity difference, while the diffusional flux term contains two parts, the first being the contribution of the presence of the particles to viscous dissipation and the second relating the same but due to size-effects. At steady state and neglecting diffusion with respect to the fluid velocity, Eq. (13) becomes

$\mu_{f} \nabla^{2} \boldsymbol{v}_{f}-\frac{\varphi \rho_{p}}{\tau}\left(\boldsymbol{v}_{f}-\boldsymbol{v}_{p}\right)+\frac{\varphi \rho_{p}}{\tau} \ell_{d}^{2} \nabla^{2}\left(\boldsymbol{v}_{f}-\boldsymbol{v}_{p}\right)=\nabla p$

with $\ell_{d}^{2}$ standing for

$\ell_{d}^{2}=\ell^{2}+\mu_{d} \frac{\tau}{\varphi \rho_{p}}$

\subsection{Relaxation time of the mass flux}

Before, moving on, we should define first this relaxation time and mean free path of the mass flux. We start with the relaxation time. Following the definitions of the phenomenological parameters (see Eqs. (5)-(6) and the text between them), we can express the relaxation time as: 
$\tau=\frac{\rho \alpha}{\chi}=\alpha \rho^{2} D T\left(\frac{\partial \eta}{\partial c}\right)^{-1}$

with $T$ the temperature and $\eta$ the difference in chemical potential of dispersion between the nanoparticles and the fluid. We need to know $\alpha$ and $\frac{\partial \eta}{\partial c}$ for finding the relaxation time. In comparison to two other thermodynamic theories (Internal Variable Theory for suspensions $[29,33,34]$ and the Generic formalism [35]), an expression equivalent to the term $\alpha$ in Eq. (11) is found. Comparing carefully the different terms for the mass flux in both the works to the ones in the present one, we come to the conclusion that $\alpha$ is equivalent to

$\alpha=\frac{1}{\rho^{2} T c(1-c)}$

The chemical potential for a nanodispersion is obtained by $\eta=\left(\frac{d g}{d c}\right)_{T, p}$, where the indices stand for a constant temperature and pressure and $g$ is the free energy of dispersion of the nanoparticles in the fluid. This is not equal to the standard free energy of mixing of blends or binary liquids, but is newly derived for dispersions and suspensions in [36]:

$\Delta g_{m, i d}=\frac{k_{B} T}{\rho_{p} V_{p}}\left((1-c) \ln (1-c)-\frac{\xi_{p f}}{2} c(1-c)\right)$

The chemical potential is then readily obtained as

$\eta=\frac{k_{B} T}{\rho_{p} V_{p}}\left(\ln \left(\frac{1}{(1-c) e}\right)+\frac{\xi_{p f}}{2}(2 c-1)\right)$,

where $V_{p}$ is the volume of one nanoparticle, $\xi_{p f} \equiv-2 \frac{z \Delta w}{k_{B} T}$ is the nanoparticle-fluid interaction parameter, with $z$ the coordination number (number of nearest lattice neighbours for both the solvent and particles), $k_{B}$ Boltzmann's constant and $\Delta w=\varepsilon_{p f}-\frac{1}{2}\left(\varepsilon_{p p}+\varepsilon_{f f}\right)$ the difference in interaction energy between like and unlike neighbours, with $\varepsilon_{p f}, \varepsilon_{p p}$ and $\varepsilon_{f f}$, respectively, the particle-fluid, particle-particle and fluid-fluid interaction energies (more information is given in [36]). The minus sign in the expression of the $\xi_{p f}$ parameter indicates that for nanofluid dispersions to be stable, this suggests an exothermic enthalpy of mixing [37]. Taking the mass fraction derivative of the chemical potential

$\frac{\partial \eta}{\partial c}=\frac{k_{B} T}{\rho_{p} V_{p}}\left(\frac{1}{(1-c)}+\xi_{p f}\right)$

gives mathematical closure for the relaxation time obtaining

$\tau=\frac{D}{c(1-c)}\left(\left(\frac{1}{(1-c)}+\xi_{p f}\right) \frac{k_{B} T}{\rho_{p} V_{p}}\right)^{-1}$.

The diffusion coefficient $D$ corresponds to the one in Eq. (7)-(8) and stands for the self-diffusion coefficient, the non-local and relaxation effects being taken into account by the time and space variations of the mass flux variable. Therefore, in case of nanoparticles suspended in a solvent, 
we can identify $D$ by the Einstein-Stokes diffusion coefficient for spherical particles $D=$ $\frac{k_{B} T}{6 \pi a_{p} \mu_{f}}$, where $a_{p}$ is the nanoparticle radius and $\mu_{f}$ the base fluid dynamic viscosity. Knowing that $V_{p}=\frac{4 \pi a_{p}^{3}}{3}$, we find the final expression for the relaxation time

$$
\tau=\tau_{p} \frac{1}{\left(\xi_{p f} c(1-c)+c\right)}
$$

where $\tau_{p}$ is defined as the well-known particle relaxation time (e.g. [38])

$$
\tau_{p}=\frac{2 \rho_{p} a_{p}^{2}}{9 \mu_{f}}
$$

\subsection{Interaction energies linked to nanolayer thickness}

Values for the interaction energies of nanoparticle dispersions are not often studied as for polymeric blends. Therefore, an interesting alternative, directly linked to the interchange energy $\Delta w$, could be the thickness of the interfacial nanolayer of dense structured fluid around the nanoparticles, which are readily available in the literature and, in absence of information, is often typically of the size $O(1) \mathrm{nm}[39,40]$. In order to find a link between the interaction energies $\varepsilon_{i j}$ and the nanolayer thickness $\ell_{i}$, we can make some observations. First, for zero overall interaction energy, i.e. $\Delta w \equiv 0$, there should not be any fluid nanolayer around the nanoparticles and the nanolayer thickness should be zero, i.e. $\ell_{i} \equiv 0$. Second, if $\Delta w<0$, than it should follow that $\ell_{i}>0$. This means that for a negative overall interaction energy (which implies $\xi_{p f}>0$ ), the particle-fluid interaction energy is smaller than the energies for the likeinteractions (it is more favourable to form links between the particles and the fluid than between like-components) and a dense fluid structure is formed around the nanoparticle. Third, if $\Delta w>$ 0 , the unlike-energy is larger than the like-ones. If the interchange energy is not too large, the nanoparticle dispersion can still be stable due to entropic contribution. In such a case, we can mathematically have that $\ell_{i}<0$. It follows that the first two cases are realistic, whilst the latter would constitute a meta-stable dispersion, where the particle-fluid repulsion is modelled by a theoretical negative nanolayer, in reality representable by the well-known concept of a slip length on the particle surface [41]. Finally, for a too large interchange energy, the stability of the dispersion is no longer guaranteed.

In order to propose a relation between the interchange energy and the interfacial nanolayer thickness, let us imagine the formation of such a nanolayer as follows. We assume that the only types of energies acting during the formation of a nanolayer, recalling that therefore $\Delta w<0$, are the Brownian surface energy and the net attractive interchange energy. The Brownian energy of a particle dispersed in a fluid equals $\frac{1}{2} k_{B} T$ per degree of freedom $[42,43]$. The formation of the interfacial nanolayer is a surface phenomenon [40], which suggests two degrees of freedom. Since, moreover, this phenomenon is related to the fluid molecules diffusing from the nanoparticle surface, the Brownian surface energy is then given by $\frac{1}{A_{p}} k_{B} T$, with $A_{p}=4 \pi a_{p}^{2}$ the nanoparticle surface. The interchange energy, in the case $\Delta w<0$, that will result into the fluid being attracted to the nanoparticle surface is given by $z \Delta w$. This interchange energy is responsible for the formation of the interfacial nanolayer, causing a difference in the surface $\Delta A_{i}=A_{i}-A_{p}$, with $A_{i}=4 \pi\left(a_{p}+\ell_{i}\right)^{2}$. The interchange surface energy is then given by $\frac{z \Delta w}{\Delta A_{i}}$. The Brownian motion, responsible for the Brownian surface energy, tends to avoid the fluid molecules to attach to the nanoparticle surface. On the other hand, the net interchange 
surface energy (in the case $\Delta w<0$ ) tends to cause an attraction of the fluid molecules towards the nanoparticle surface. The sum of the surface energies, the net surface energy $\Delta E_{S}=\frac{z \Delta w}{\Delta A_{i}}+$ $\frac{1}{A_{p}} k_{B} T$, will govern the process, depicted in Fig. 2.
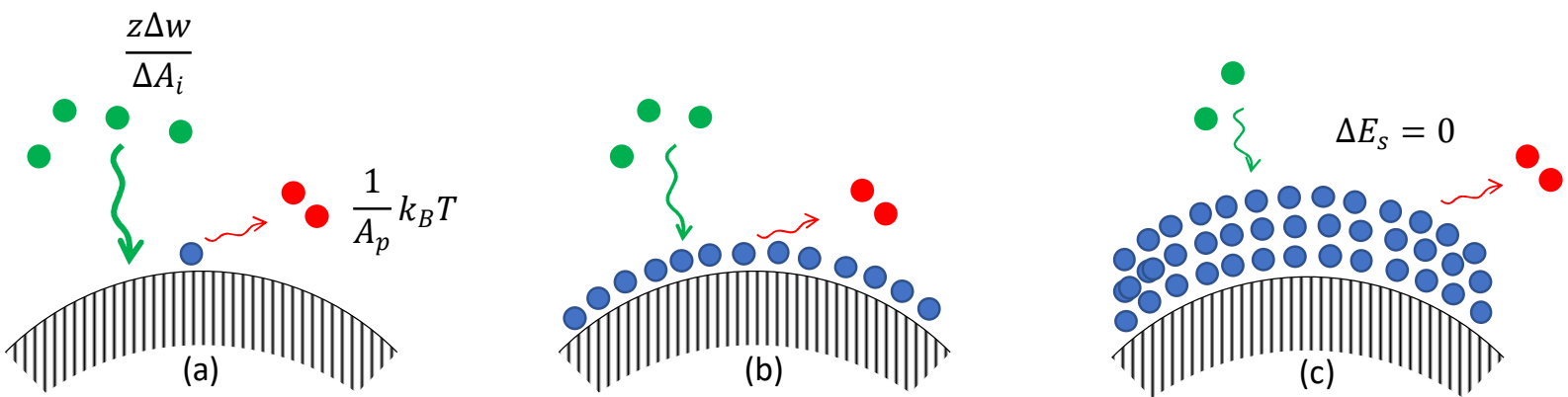

Figure 2: Competition between the Brownian and interchange surface energies governing the thickness of the interfacial nanolayer: (a) the interchange energy is more important than the Brownian surface energy in a way that it is favourable for the fluid particles to attach to the particle substrate, (b) as the substrate is being covered by dense fluid layers, the interchange energy weakens, until (c) it is equal to the Brownian surface energy leading to a stable interfacial nanolayer

At the beginning of the formation of the interfacial nanolayer, $\Delta E_{s}<0$. As long as this is the case, the interfacial nanolayer continues to grow. However, each layer of fluid adding up in this nanolayer will decrease the attraction between the nanoparticle surface and the fluid molecules and $\Delta E_{s}$ increases. When the interfacial nanolayer thickness is sufficiently large, the interchange surface energy will equal the Brownian surface energy, reaching an equilibrium situation, $\Delta E_{s}=0$ or $\frac{1}{A_{p}} k_{B} T+\frac{z \Delta w}{\Delta A_{i}}=0$. The relation between the interchange energy and the interfacial nanolayer thickness is then given by

$\Delta w=-\frac{1}{z}\left(\left(1+\frac{e_{i}}{a_{p}}\right)^{2}-1\right) k_{B} T$

The interaction parameter is then finally given by

$\xi_{p f}=2\left(\left(1+\frac{\ell_{i}}{a_{p}}\right)^{2}-1\right)$

The interfacial nanolayer thickness can be obtained in several ways. One of them is in the experimental way by means of e.g. reflective X-ray, nano-ultrasonics, nuclear magnetic resonance studies [44-46], or by using molecular dynamics [47-49]. Another way is based on the electron density profile at the interface applied to a solid-liquid system, where the expression $\delta=\sqrt{2 \pi} \sigma$ is proposed, with $\delta$ an average liquid-layer thickness and $\sigma$ a characteristic length related to the diffuseness of the interfacial boundary, leading to a typical value that falls in the range $0.4-0.6 \mathrm{~nm}$, from which follows that $\delta$ is of the order of $1-1.5$ $\mathrm{nm}[50,51]$. As a final option for $\ell_{i}$, we can take for illustration the values $\ell_{i}=1-1.5 \mathrm{~nm}$. Such an approximation can be useful when no value for the interfacial nanolayer thickness is at hand. Both the aforementioned methods will be used in our model for the effective viscosity.

\subsection{Relative velocity}


In Eqs. (10) and (11), two unknowns are still present, the extra viscosity factor $\mu_{D}$ and the relative velocity $\boldsymbol{v}_{f}-\boldsymbol{v}_{p}$. The relation between the nanoparticle and fluid velocities is established by assuming that the motion of the spherical nanoparticles of mass $m_{p}$ is essentially due to the presence of a Stokes force $\boldsymbol{F}_{s}$, in interaction with a buoyancy force $\boldsymbol{F}_{\boldsymbol{b}}$, caused by a pressure gradient. First, we can say that for one nanoparticle, the force balance is given by

$m_{p} \frac{d v_{p}}{d t}=\boldsymbol{F}_{b}+\boldsymbol{F}_{s}$

where $m_{p}$ is the mass of one nanoparticle [38,52]. The same type of equation is proposed in [53] However, the nanoparticles in motion also exerce a force $-n_{p}\left(\boldsymbol{F}_{b}+\boldsymbol{F}_{s}\right)$ on the fluid, which undergoes a total driving force $\boldsymbol{F}_{\boldsymbol{d}}$ due to the pressure gradient as well $[38,53]$. The force balance on the fluid is then given by

$m_{f} \frac{d v_{f}}{d t}=\boldsymbol{F}_{\boldsymbol{d}}-n_{p}\left(\boldsymbol{F}_{b}+\boldsymbol{F}_{s}\right)$

Here the force of the nanoparticles acting on the fluid is multiplied by $n_{p}$ to account for all the nanoparticles. The total driving force [38] is given by

$\boldsymbol{F}_{\boldsymbol{d}}=-V_{t} \nabla p$

while that on one nanoparticle [38] writes

$\boldsymbol{F}_{b}=-V_{p} \nabla p$

The Stokes drag force on one spherical nanoparticle is well-known $[38,52,54,55]$ and given by

$\boldsymbol{F}_{s}=6 \pi a_{p} \mu_{f}\left(\boldsymbol{v}_{\boldsymbol{f}}-\boldsymbol{v}_{\boldsymbol{p}}\right)$

Filling (28)-(30) in (27) and rearranging gives the force balance for the fluid in interaction with the nanoparticles as

$m_{f} \frac{d v_{f}}{d t}=-\left(V_{t}-n_{p} V_{p}\right) \nabla p-n_{p} 6 \pi a_{p} \mu_{f}\left(\boldsymbol{v}_{f}-\boldsymbol{v}_{\boldsymbol{p}}\right)$

Since we operate in the framework of a porous-like configuration, we can assume a local Darcy law, where the pressure gradient gives rise to

$-\nabla p=\frac{\mu_{f}}{K} \boldsymbol{v}_{f}$

wherein $K$ designates the local permeability expressed by [32]

$K=\frac{\tau \mu_{f}(1-\varphi)}{\varphi \rho_{p}}$ 
Filling (32)-(33) into (31), rearranging the terms and, to be consistent with (12)-(14), we evaluate at steady-state fluid flow, which leads finally to

$$
\left(\boldsymbol{v}_{f}-\boldsymbol{v}_{\boldsymbol{p}}\right)=\left(\xi_{p f} c(1-c)+c\right) \boldsymbol{v}_{\boldsymbol{f}}
$$

Here we see that the relative velocity depends on the volume fraction, the densities of the nanoparticles (or nanodroplets in case of a stabilized emulsion) and the fluid-particle interaction.

\subsection{Extra viscosity coefficient}

As for $\mu_{d}$, we go back to the viscous stress tensor in Eq. (11). With (34), we can rewrite (11) into

$\boldsymbol{\sigma}=\mu_{f} \nabla \boldsymbol{v}_{f}+\mu_{d}\left(\xi_{p f} c(1-c)+c\right) \nabla \boldsymbol{v}_{f}$

A similar derivation is performed in a different context in references [56-58], proposing a viscous stress tensor $\boldsymbol{\sigma}_{\boldsymbol{d} d}$ : these papers deal with particles (in a strongly diluted suspension, i.e. $\varphi \ll 1$, with no density effects, i.e. $\rho_{p} \equiv \rho_{f}$ and $c \equiv \varphi$, hard spheres without any type of interactions, i.e. $\xi_{p f} \equiv 0$, and no influence of other particles) with a dipole strength that are shown to give rise to an extra viscous contribution of the form

$\boldsymbol{\sigma}_{\boldsymbol{d d}}=\mu_{f} \nabla \boldsymbol{v}_{f}+\frac{5}{2} \varphi \mu_{f} \nabla \boldsymbol{v}_{f}$

Comparing (36) to (35), this gives

$\mu_{d} \equiv \frac{5}{2} \mu_{f}$

The viscous stress tensor becomes

$\boldsymbol{\sigma}=\mu_{f}\left(1+\frac{5}{2}\left(c+\xi_{p f} c(1-c)\right)\right) \nabla \boldsymbol{v}_{f}$

\subsection{Mean free path of the mass flux}

The mean free path $\ell$ in Eq. (15) is that of the mass flux, which is an unmeasured quantity. As we related the mass flux relaxation time to the particle relaxation time, we will do the same for the mean free path. Brownian kinetics predict the diffusion coefficient of a particle in a fluid (assuming that the particles behave as a gas in this framework) as $D \propto \frac{\ell_{p}^{2}}{\tau_{p}}$ [33]. From Eq. (8), one can suggest that $D \propto \frac{\ell^{2}}{\tau}$. As $D$ is the self-diffusion coefficient, we should make this evaluation without particle-fluid interaction (i.e. $\xi_{p f} \equiv 0$ ) and for $\rho_{p}=\rho_{f}$. With these conditions and using Eq. (22), we can easily obtain that

$$
\ell^{2}=\frac{\ell_{p}^{2}}{\varphi}
$$

\subsection{Final description of the nanofluid dynamics}


Having developed expressions for the relaxation time $\tau$, the extra viscosity coefficient $\mu_{d}$, the mass flux mean free path $\ell$, and the relative velocity, we can rewrite Eqs. (14) and (15) in the known terms and one variable $\boldsymbol{v}_{f}$ :

$\mu_{f}\left(1+\frac{9}{2} K n_{p}^{2}(c+\Xi)^{2}+\frac{5}{2}(c+\Xi)\right) \nabla^{2} \boldsymbol{v}_{f}-\mu_{f} \frac{9}{2 a_{p}^{2}} \varphi(c+\Xi)^{2} \boldsymbol{v}_{f}=\nabla p$

with

$\Xi=2\left(\left(1+\Sigma_{i}\right)^{2}-1\right) c(1-c)$

$\Sigma_{i}=\frac{\ell_{i}}{a_{p}}$

$K n_{p}=\frac{\ell_{p}}{a_{p}}$

\subsection{Cylindrical-pore approximation}

Eq. (40) is a general equation. As we describe the viscous behaviour of the nanoparticle dispersion as the interaction between the nanoparticles and the fluid flowing in between them, we need to make some specifications. Imagine now that the base fluid is percolating in between the nanoparticles, by similarity with a fluid flowing through a porous medium. As such, the fluid is assumed to move through a cylindrical cross section, with a radius equivalent to the hydraulic radius $R$, bounded by a wall, equivalent to a flat lining of the nanoparticles. This analogy is depicted in Fig. 3.
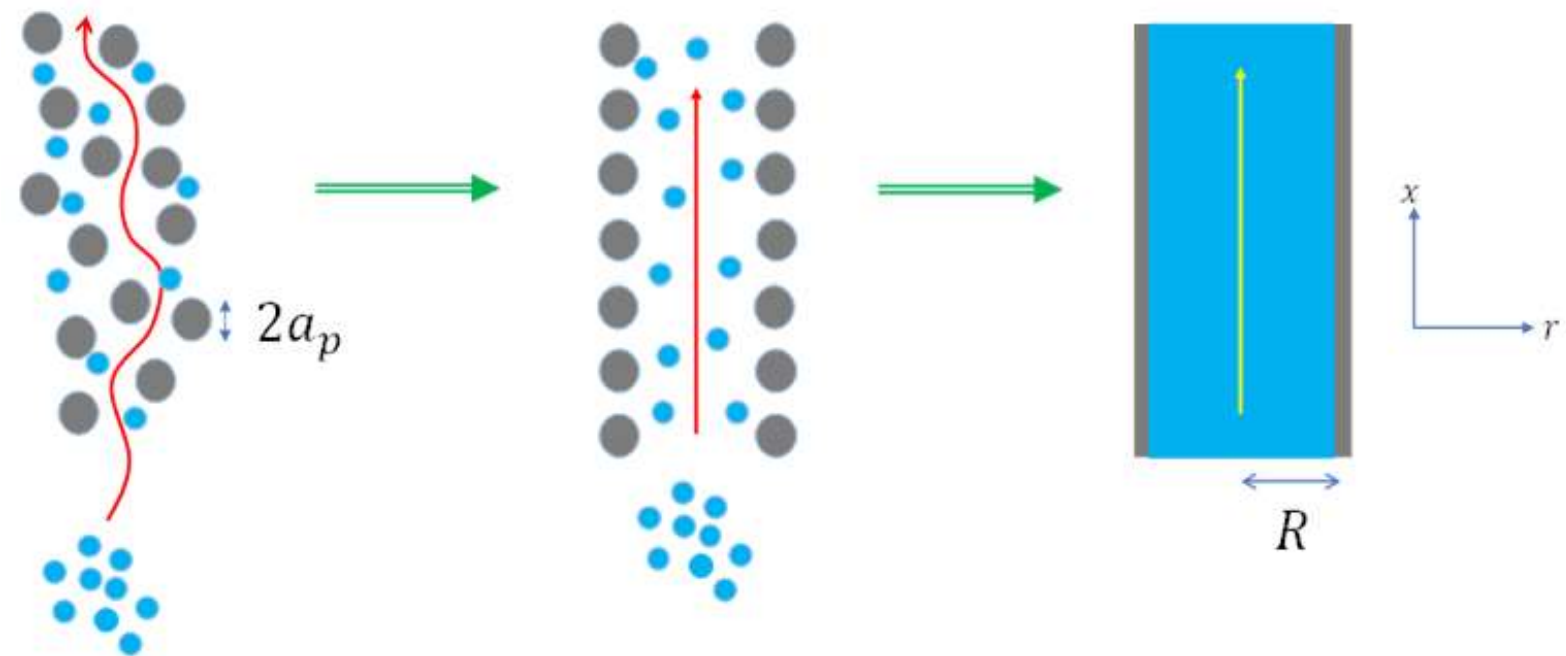

Figure 3: Schematic representation of the porous description of fluid flow in stable nanodispersions and its equivalent developed tube flow with hydraulic radius $R$.

In such a configuration, the fluid velocity becomes one-dimensional, being described by the $x$ component, where $x$ is the coordinate in the flow direction. Flowing in between the aforementioned walls the fluid velocity is assumed to vary only radially as a function of the $r$ coordinate, so that $\boldsymbol{v}_{f}=v_{f}(r)$. The pressure is assumed to vary only across the flow direction. Note that the variables are now all one-dimensional and are no longer written in vector form. Eq. (40) will then be used to describe the fluid flow through a cylindrical cross section, with a radius $r$ varying from 0 to $R$. In presence of porous structures, the hydraulic radius, $R=$ $2 V_{f} / A_{f}$, is usually defined $[59,60]$ as the volume $V_{f}$ open to flow divided by half the total wetted 
surface $A_{f}$. Since the total volume $V_{t}$ is the volume $V_{f}$ occupied by the fluid plus the total volume $n_{p} V_{p}$ of the particles ( $n_{p}$ is the number of spherical particles of volume $V_{p}$ each), one may write

$V_{t}=V_{f}+n_{p} V_{p}$

and, in virtue of the definition of the volume fraction of nanoparticles $\varphi=n_{p} V_{p} / V_{t}$, it follows from (12) that

$V_{f}=V_{t}(1-\varphi)$

The total wetted surface, $A_{f}$, equals the number of spherical particles, $n_{p}$ times its surface, $A_{p}$, i.e. $A_{f}=n_{p} A_{p}$, whereas the number of spherical particles equals the volume of all particles, $V_{t} \varphi$ divided by the volume of one particle, $V_{p}$, i.e. $n_{p}=V_{t} \varphi / V_{p}$. The hydraulic radius may therefore be given the form

$R=2 \frac{V_{t}(1-\varphi)}{n_{p} A_{p}}=2 \frac{V_{p}}{A_{p}} \frac{(1-\varphi)}{\varphi}=\frac{2 a_{p}}{3} \frac{1-\varphi}{\varphi}$

after use of the expressions for the volume and surface of the spherical nanospheres, $a_{p}$ standing for the radius of the particles. Using such a description, Eq. (40) is then written as

$\mu_{f}\left(1+\frac{9}{2} K n_{p}^{2}(c+\Xi)^{2}+\frac{5}{2}(c+\Xi)\right) \frac{1}{r} \frac{\partial}{\partial r}\left(r \frac{\partial v_{f}}{\partial r}\right)-\mu_{f} \frac{9}{2 a_{p}^{2}} \varphi(c+\Xi)^{2} v_{f}=\frac{\partial p}{\partial x}$,

with a no-slip condition:

$v_{f}(r=R)=0$.

This boundary condition is justified as the interaction between the nanoparticles and the fluid is given by the interaction parameter, assuming that the influence of the dense interfacial nanolayer on the velocity profile is negligible. This assumption is especially justified when one notices that for volume fractions typical for nanoparticle dispersions $R \gg \ell_{i}$. The second boundary condition expresses that the velocity is maximum at the centre of the cylinder:

$\left.\frac{\partial v_{f}}{\partial r}\right|_{r=0}=0$.

\subsection{Adaptation for non-spherical particles}

The deduction performed above is under the assumption that the particles are spherical or close to spherical. For cylindrical particles of which the length is of the order of their radius or for square-like particles, they can often be approximated as spherical-like particles. However, if the length $L_{p}$ of the cylindrical particles is much larger than the cylinder-radii $a_{p}$ of those particles, an adaptation is needed. Three changes are in order here. One in relation with the drag force, leading to a cylindrical-particle relaxation time $\tau_{p, c}$, the other in relation with the interfacial nanolayer and the third in relation with the hydraulic radius. It has been proposed that for a cylindrical particle the drag force in Eq. (30) should be given by [61] 
$\boldsymbol{F}_{s}=6 \pi \mu_{f}\left(\frac{1}{3} a_{n}+\frac{2}{3} a_{s}\right)\left(\boldsymbol{v}_{\boldsymbol{f}}-\boldsymbol{v}_{\boldsymbol{p}}\right)$

where $a_{n}$ is the radius of a sphere whose projection has an equal surface of that of the object projected normal to the flow direction and $a_{s}$ is the radius of a sphere having the same surface as that of the object on which friction occurs. For a cylindrical particle of which the axis is in the flow direction, these would be $a_{n, c}=\frac{2}{\pi} L_{p}$ and $a_{s, c}=\frac{L_{p}}{2}$. For a particle volume of $V_{p}=$ $\pi a_{p}^{2} L_{p}$, the cylindrical-particle relaxation time becomes $\tau_{p, c}=\frac{2 \rho_{p} a_{p}^{2}}{9 \mu_{f}}\left(\frac{9}{4} \frac{\pi}{2+\pi}\right)$. Comparing with Eq. (23), this means that for a cylindrical particle the radius $a_{p}$, in the expressions depending on $\tau_{p, c}$, should be replaced as

$a_{p} \rightarrow a_{p} \sqrt{\frac{9}{4} \frac{\pi}{2+\pi}}$

while for the interfacial nanolayer the interaction parameter $\xi_{p f, c}$ would rather be given, following the definitions in section 2.3 , by

$\xi_{p f, c}=2 \frac{\ell_{i}}{a_{p}}$

and the hydraulic diameter, following the definitions in section 2.8 , would be given by

$R_{C}=2 \frac{V_{p}}{A_{p}} \frac{(1-\varphi)}{\varphi}=a_{p} \frac{1-\varphi}{\varphi}$

Some remarks are in place here. It should be noted that the shape of the particle does not influence the development of the mass flux relaxation time via the chemical potential, since the difference is incorporated in the particle relaxation time. Together with the interaction parameter, they are responsible for the influence of the particle size in the velocity equation, whilst the length of the cylindrical particles does not play a role here. Moreover, it is interesting to remark that the development for cylindrical particles will lead to a particle radius that equals $a_{p} \sqrt{\frac{9}{4} \frac{\pi}{2+\pi}} \approx 1,17 a_{p}$, which suggests that, as approximation, the model for spherical particles would be a good estimate.

\section{Effective viscosity for nanodispersions}

\subsection{Full model}

Let us, for the sake of compactness, define

$$
\begin{aligned}
& A_{1}=\frac{9}{2} K n_{p}^{2}(c+\Xi)^{2}+\frac{5}{2}(c+\Xi), \\
& A_{2}=\frac{9}{2 a_{p}^{2}} \varphi(c+\Xi)^{2}
\end{aligned}
$$

so that relation (47) reads as

$$
\left(1+A_{1}\right) \frac{1}{r} \frac{\partial}{\partial r}\left(r \frac{\partial v_{f}}{\partial r}\right)-A_{2} v_{f}=\frac{1}{\mu_{f}} \frac{\partial p}{\partial x}
$$


Having solved equation (56) coupled to the boundary conditions (48) and (49) for the velocity profile $v_{f}(r)$, we can determine its mean value $\left.\left\langle v_{f}\right\rangle=\frac{1}{\pi R^{2}} \int_{0}^{R} 2 \pi r v_{f}(r) d r\right)$, which is given by

$$
\left\langle v_{f}\right\rangle=-\frac{1}{\mu_{f} A_{2}} \frac{\partial p}{\partial x}\left(1-\frac{{ }_{0} \mathcal{F}_{1}\left(2, \frac{A_{2} R^{2}}{4+4 A_{1}}\right)}{{ }_{0} \mathcal{F}_{1}\left(1, \frac{A_{2} R^{2}}{4+4 A_{1}}\right)}\right)
$$

where ${ }_{0} \mathcal{F}_{1}(n, x)$ is the $0 / 1$ regularized hypergeometric function with parameters $n$ and $x$. We recall that our final goal is to determine an expression of the effective nanofluid viscosity. This is achieved by comparing the results (37)-(38) with the mean velocity measured in a Poiseuillelike flow with a viscosity $\mu_{n f}$. In other words, we assume that the mean fluid velocity is the same as that of Poiseuille flow in a cylindrical pipe with radius $R$, with an effective viscosity $\mu_{n f}$ that accounts for the presence of nanoparticles. The corresponding mean fluid velocity is the classical result

$$
\left\langle v_{f}\right\rangle=-\frac{R^{2}}{8 \mu_{n f}} \frac{\partial p}{\partial x}
$$

We can then readily seen that the effective viscosity is given by

$\mu_{n f}=\frac{A_{2} R^{2} \mu_{f}}{8}\left(1-\frac{{ }_{0} \mathcal{F}_{1}\left(2, \frac{A_{2} R^{2}}{4+4 A_{1}}\right)}{{ }_{0} \mathcal{F}_{1}\left(1, \frac{A_{2} R^{2}}{4+4 A_{1}}\right)}\right)^{-1}$

which is valid for both spherical and cylindrical nanoparticles. The difference lies in the aforementioned definitions for $R, \xi_{p f}$ and $\tau_{p}$. Using these definitions in Eq. (59) will finally result into the effective viscosity of spherical nanoparticle dispersions, given by

$\mu_{n f}=\mu_{f} \frac{\left(\frac{\varphi \rho_{p}}{\rho}+\Xi_{S}\right)^{2}(1-\varphi)^{2}}{4 \varphi}\left(1+\frac{{ }_{0} \mathcal{F}_{1}\left(2, X_{S}\right)}{{ }_{0} \mathcal{F}_{1}\left(1, X_{S}\right)-{ }_{0} \mathcal{F}_{1}\left(2, X_{S}\right)}\right)$

where

$$
\begin{aligned}
& X_{s}=\frac{\left(\frac{\varphi \rho_{p}}{\rho}+\Xi_{s}\right)^{2}(1-\varphi)^{2}}{\varphi\left(2+2\left(\frac{5}{2}\left(\frac{\varphi \rho_{p}}{\rho}+\Xi_{s}\right)+\frac{9}{2} K n_{p, s}^{2}\left(\frac{\varphi \rho_{p}}{\rho}+\Xi_{s}\right)^{2}\right)\right)} \\
& \Xi_{s}=2\left(\left(1+\frac{\ell_{i}}{a_{p, s}}\right)^{2}-1\right) \frac{\varphi \rho_{p}}{\rho}\left(1-\frac{\varphi \rho_{p}}{\rho}\right) \\
& K n_{p, s}=\frac{\ell_{p}}{a_{p, s}}
\end{aligned}
$$

where $a_{p, s}$ is a spherical radius. For cylindrical nanoparticle dispersions, we obtain the following effective viscosity

$$
\mu_{n f}=\mu_{f} \frac{\left(\frac{\varphi \rho_{p}}{\rho}+\Xi_{c}\right)^{2}(1-\varphi)^{2}}{4\left(\frac{\pi}{2+\pi}\right) \varphi}\left(1+\frac{{ }_{0} \mathcal{F}_{1}\left(2, X_{C}\right)}{{ }_{0} \mathcal{F}_{1}\left(1, X_{C}\right)-{ }_{0} \mathcal{F}_{1}\left(2, X_{C}\right)}\right)
$$


where

$$
\begin{aligned}
& X_{c}=\frac{\left(\frac{\varphi \rho_{p}}{\rho}+\Xi_{c}\right)^{2}(1-\varphi)^{2}}{\frac{\pi}{2+\pi} \varphi\left(2+2\left(\frac{5}{2}\left(\frac{\varphi \rho_{p}}{\rho}+\Xi_{c}\right)+\frac{9}{2} K n_{p, c}^{2}\left(\frac{\varphi \rho_{p}}{\rho}+\Xi_{c}\right)^{2}\right)\right)} \\
& \Xi_{c}=2 \frac{\ell_{i}}{a_{p, c}} \frac{\varphi \rho_{p}}{\rho}\left(1-\frac{\varphi \rho_{p}}{\rho}\right) \\
& K n_{p, c}=\frac{\ell_{p}}{\frac{3}{2} \sqrt{\frac{\pi}{2+\pi}} a_{p, c}}
\end{aligned}
$$

where $a_{p, c}$ is a cylindrical radius. The radii $a_{p, s}$ and $a_{p, c}$ will be written in the foregoing as $a_{p}$ indicating clearly whether it concerns a spherical or cylindrical radius.

\subsection{Asymptotic cases}

In case $\nabla^{2} v_{f} \gg v_{f} / R^{2}$, the mean value for the fluid velocity simplifies as

$$
\left\langle v_{f}\right\rangle=-\frac{1}{8 \mu_{f}} \frac{\partial p}{\partial x} \frac{R^{2}}{1+A_{1}}
$$

Comparison between (68) and (58) yields the simplified expression for the effective viscosity for spherical nanoparticle dispersions to be

$\mu_{n f}=\mu_{f}\left(1+\frac{5}{2}\left(\frac{\varphi \rho_{p}}{\rho}+\Xi_{s}\right)+\frac{9}{2} K n_{p, s}^{2}\left(\frac{\varphi \rho_{p}}{\rho}+\Xi_{s}\right)^{2}\right)$

where $\Xi_{s}$ and $K n_{p, s}$ are given by (62) and (63), respectively. For cylindrical nanoparticle dispersions, the simplified effective viscosity is then given by

$\mu_{n f}=\mu_{f}\left(1+\frac{5}{2}\left(\frac{\varphi \rho_{p}}{\rho}+\Xi_{c}\right)+\frac{9}{2} K n_{p, c}^{2}\left(\frac{\varphi \rho_{p}}{\rho}+\Xi_{c}\right)^{2}\right)$

where $\Xi_{c}$ and $K n_{p, c}$ are given by (66) and (67), respectively. For engineering applications and material functions for numerical modelling a simple expression is often needed. By analysing Eqs. (69) and (70), we can mention that often for nanoparticle dispersions in liquids, $K n_{p, s} \ll$ 1 and $K n_{p, c} \ll 1$. This leads to the following simplifications

$$
\begin{aligned}
& \mu_{n f}=\mu_{f}\left(1+\frac{5}{2}\left(\frac{\varphi \rho_{p}}{\rho}+2\left(\left(1+\frac{\ell_{i}}{a_{p, s}}\right)^{2}-1\right) \frac{\varphi \rho_{p}}{\rho}\left(1-\frac{\varphi \rho_{p}}{\rho}\right)\right)\right) \\
& \mu_{n f}=\mu_{f}\left(1+\frac{5}{2}\left(\frac{\varphi \rho_{p}}{\rho}+2 \frac{\ell_{i}}{a_{p, c}} \frac{\varphi \rho_{p}}{\rho}\left(1-\frac{\varphi \rho_{p}}{\rho}\right)\right)\right)
\end{aligned}
$$

for spherical and cylindrical nanoparticles, respectively. For nanoparticle with larger sizes or for weak particle-fluid interaction, both of which lead to $\ell_{i} \ll a_{p}$, the effective viscosity is given by a Einstein-like expression, corrected for density dependency:

$$
\mu_{n f}=\mu_{f}\left(1+\frac{5}{2} \frac{\varphi \rho_{p}}{\rho}\right)
$$


If, furthermore, the densities of the nanoparticles and the fluid are close to one another, i.e. $\rho_{p} \approx \rho_{f} \approx \rho$, Einstein's equation for the viscosity of dispersion is found back

$$
\mu_{n f}=\mu_{f}\left(1+\frac{5}{2} \varphi\right)
$$

\section{Results and discussion}

\subsection{Material properties}

Table 1 reproduces these material properties for several nanofluids. The base fluids considered in this study are water (W) and ethylene-glycol (EG) respectively. The nanoparticles are selected as alumina $\left(\mathrm{Al}_{2} \mathrm{O}_{3}\right)$, magnetite $\left(\mathrm{Fe}_{3} \mathrm{O}_{4}\right)$, silicon-carbide $(\mathrm{SiC})$, carbon nanotube $(\mathrm{CNT})$, graphite (GR) and nanodiamonds (ND). The values are taken at a reference temperature $T_{r e f}$, corresponding to the one at which the experiments were performed. The fluid densities are wellknown standard values, which can be found in chemical handbooks. The mean free path is taken to be of the order of magnitude of the equivalent diameter of the fluid molecule, around 0.38 $\mathrm{nm}$ for water and 0.56 for ethylene glycol. Wherever a value is non-standard, it is mentioned. The particle densities are assumed to be negligibly dependent on the temperature for the temperature range used in this work $\left(10-60{ }^{\circ} \mathrm{C}\right)$, which is also the typical range of operating temperatures for nanodispersions. Other parameters are the volume fraction $\varphi$ and the nanoparticle radius $a_{p}$.

Table 1: Material properties of nanodispersions

\begin{tabular}{|l|l|l|l|l|l|l|l|}
\hline Nanofluids & $\begin{array}{l}T_{r e f} \\
{\left[{ }^{\circ} \mathrm{C}\right]}\end{array}$ & $\begin{array}{l}\rho_{f} \\
{\left[\mathrm{~kg} / \mathrm{m}^{3}\right]}\end{array}$ & $\begin{array}{l}\rho_{p} \\
{\left[\mathrm{~kg} / \mathrm{m}^{3}\right]}\end{array}$ & $\begin{array}{l}\ell_{i} \\
{[\mathrm{~nm}]}\end{array}$ & $\begin{array}{l}\ell_{p} \\
{[\mathrm{~nm}]}\end{array}$ & $\begin{array}{l}2 a_{p} \\
{[\mathrm{~nm}]}\end{array}$ & Ref. \\
\hline $\mathrm{Fe}_{3} \mathrm{O}_{4}-$ water & 20 & 998 & 5170 & 0.95 & 0.38 & 25 & {$[14]$} \\
\hline $\mathrm{SiC}-40 \mathrm{EG} / 60 \mathrm{~W}$ & 10 & 1049 & 3510 & 1.23 & 0.49 & 30 & {$[15]$} \\
\hline $\mathrm{Al}_{2} \mathrm{O}_{3}-\mathrm{EG}$ & 10 & 1123 & 3950 & 0.9 & 0.56 & 43 & {$[16]$} \\
\hline $\mathrm{Al}_{2} \mathrm{O}_{3}-\mathrm{EG}$ & 10 & 1123 & 3950 & 0.9 & 0.56 & 8 & {$[16]$} \\
\hline $\mathrm{GR}-$ water & 60 & 983 & 2267 & 0.9 & 0.38 & 8 & {$[17]$} \\
\hline $\mathrm{Al}_{2} \mathrm{O}_{3}-$ water & 25 & 997 & 3950 & 1.2 & 0.38 & 25 & {$[18]$} \\
\hline $\mathrm{ND}-$ alkalinated water & 40 & 992 & 3100 & 1.5 & 0.38 & 10 & {$[19]$} \\
\hline CNT - distilled water & $10 \& 20$ & $1000 \&$ & 1800 & 1.2 & 0.38 & 9 & {$[20]$} \\
\hline
\end{tabular}

${ }^{\mathrm{a}}$ Reported value for alkalinated water in [32]

The values for $\ell_{i}$ need some comments. For $\mathrm{Al}_{2} \mathrm{O}_{3}$ - water nanofluids, nano-ultrasonics [45] and molecular dynamics [62] studies show that bulk values for the water density and viscosity are measured at a distance around $1 \mathrm{~nm}$ from the $\mathrm{Al}_{2} \mathrm{O}_{3}$ surface. Nuclear magnetic resonance[46] shows the presence of a thin ordered layer of water molecules of about $1.4 \mathrm{~nm}$. We took the mean value of $1.2 \mathrm{~nm}$. In [63], it was reported that the thickness of the nanolayer around alumina nanoparticles in water was somewhat larger than that in ethylene glycol, the latter of which would be around 1.6 the equivalent diameter, i.e. of the order of $0.9 \mathrm{~nm}$. As for the CNT - distilled-water nanofluid, molecular dynamics [64] predicts a density attaining the mean value of that of water outside a layer thickness of $1.2 \mathrm{~nm}$. For a graphene - water nanofluid the same molecular dynamics study shows a value of $0.9 \mathrm{~nm}$ [64], confirmed by potential-energy profiles [65]. Neglecting the effect of inner graphite layers, we assume that the same layer thickness would occur for the graphite - water nanodispersion, i.e. $0.9 \mathrm{~nm}$. For the $\mathrm{Fe}_{3} \mathrm{O}_{4}-\mathrm{W}$ 
and $\mathrm{SiC}-40 \mathrm{EG} / 60 \mathrm{~W}$ nanofluids, we could not find recorded or precise measured layer thicknesses. As mentioned above and resulting from molecular dynamics calculations, often values of the order of $1 \mathrm{~nm}$ are taken. Due to lack of specific information, we to find it reasonable to assume a liquid layer of thickness $1 \mathrm{~nm}$ for these two nanofluids. The ND particles form stable nanosuspensions, reported to have a water interfacial layer going from around $1 \mathrm{~nm}$ and up to 4 molecular equivalent diameters, which corresponds to $1.5 \mathrm{~nm}[66,67]$. The ND particles in [19] have a size of $10 \mathrm{~nm}$, whereas X-ray diffraction indicates a size of $11.4 \mathrm{~nm}$. This tends to favour the aforementioned value of $1.5 \mathrm{~nm}$ as the interfacial layer. Due to lack of specific information on the nanolayer thickness of a $\mathrm{SiC}-40 \mathrm{EG} / 60 \mathrm{~W}$ nanodispersion, observing from the above findings from the literature that the nanolayer thickness is often roughly between 2 and 3 times the equivalent diameter. Therefore, we assume 2.5 times the equivalent diameter of a $40 \mathrm{EG} / 60 \mathrm{~W}$ fluid, leading to $1.23 \mathrm{~nm}$. The same is done for the $\mathrm{Fe}_{3} \mathrm{O}_{4}$ - water nanodispersion, leading to a nanolayer thickness of $0.95 \mathrm{~nm}$, being close to the value for the $\mathrm{Al}_{2} \mathrm{O}_{3}$ - water system, which seems to be a realistic value.

\subsection{Comparison with other models}

The model proposed in the previous section, Eqs. (60)-(63) for the spherical nanoparticles and Eqs. (64)-(67) for the cylindrical ones, will be compared to experimental data, referenced in Table 1. For comparison, we also traced our model in the asymptotic behaviour of a zero-flux, neglecting thereby the rarity of the base fluid, i.e. Eqs. (71) and (72) for, respectively, spherical and cylindrical nanoparticles. Finally, we compared with a mass- and volume-fractiondependent Einstein equation, i.e. Eqs. (73) and (74), respectively. For all these models, the material properties are taken from Table 1. Fig. 4.1 shows these results for two cases, i.e. 43 and $8 \mathrm{~nm}$ diameter $\mathrm{Al}_{2} \mathrm{O}_{3}$ nanoparticles dispersed in ethylene glycol.
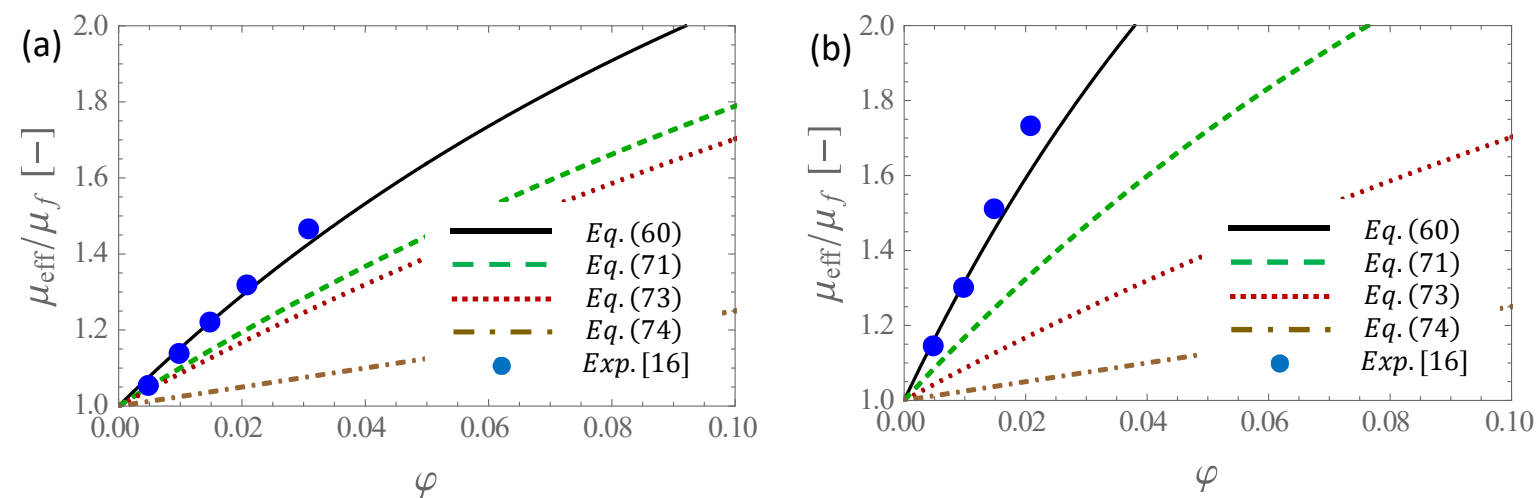

Figure 4: Relative effective viscosity of (a) $43 \mathrm{~nm} \mathrm{Al}{ }_{2} \mathrm{O}_{3}$ nanoparticles and (b) $8 \mathrm{~nm} \mathrm{Al}_{2} \mathrm{O}_{3}$ nanoparticles, both dispersed in ethylene glycol.

Fig. 4 clearly shows that a smaller nanoparticle diameter results, the only different parameter, into a considerably higher effective viscosity. From our model, we can see that the size of the nanoparticles intervenes in two terms, one in relation with $\ell_{i}$ and the second in relation with $\ell_{p}$. As, generally for liquid basefluids, $\ell_{p} \ll a_{p}$, the second term can be safely neglected. However, when $a_{p}=O\left(\ell_{i}\right)$ (note that the case $a_{p}<\ell_{i}$ leads for liquid base fluids generally to atomic scales, and therefore is not relevant for the present discussion), the effect of the nanoparticle size becomes relevant. The nanoparticle-size dependence of the effective viscosity follows $\frac{\ell_{i}}{a_{p}}$, as our model shows. The observation that a smaller size leads to a higher viscosity, can here be explained by the strength of the particle-fluid interaction, represented here by $\ell_{i}$. 
The smaller the nanoparticle is, for a given volume fraction, the higher the particle-fluid interaction surface is, the higher the contribution of this interaction is for increasing the effective viscosity. It is then clear that for a diameter of $8 \mathrm{~nm}$, i.e. $a_{p}=4 \mathrm{~nm}$, and for $\ell_{i}=0.9$ $\mathrm{nm}$, the effective viscosity is higher than for the nanodispersion with $43 \mathrm{~nm}$ nanoparticles. Such a tendency is largely observed in the literature, e.g. in [18,20,28,30,68-70], and our model explains this via the particle-fluid interaction that finds its source in the chemical potential of dispersion (Eq. (19), (24), (25) and (52)). Fig. 4.1 also shows that neglecting the effect of the flux would give a reasonable order of magnitude comparison with respect to the experimental data, especially at low volume fractions. The mass-fraction-dependent Einstein equation starts to deviate considerably, whilst the classical one is, as expected, far from the experimental data. Another interesting comparison that can be made is the comparison between two nanodispersions of $25 \mathrm{~nm}$ nanoparticles dispersed in water, where one nanoparticle is $\mathrm{Fe}_{3} \mathrm{O}_{4}$ and the other $\mathrm{Al}_{2} \mathrm{O}_{3}$, presented in Fig. 5.
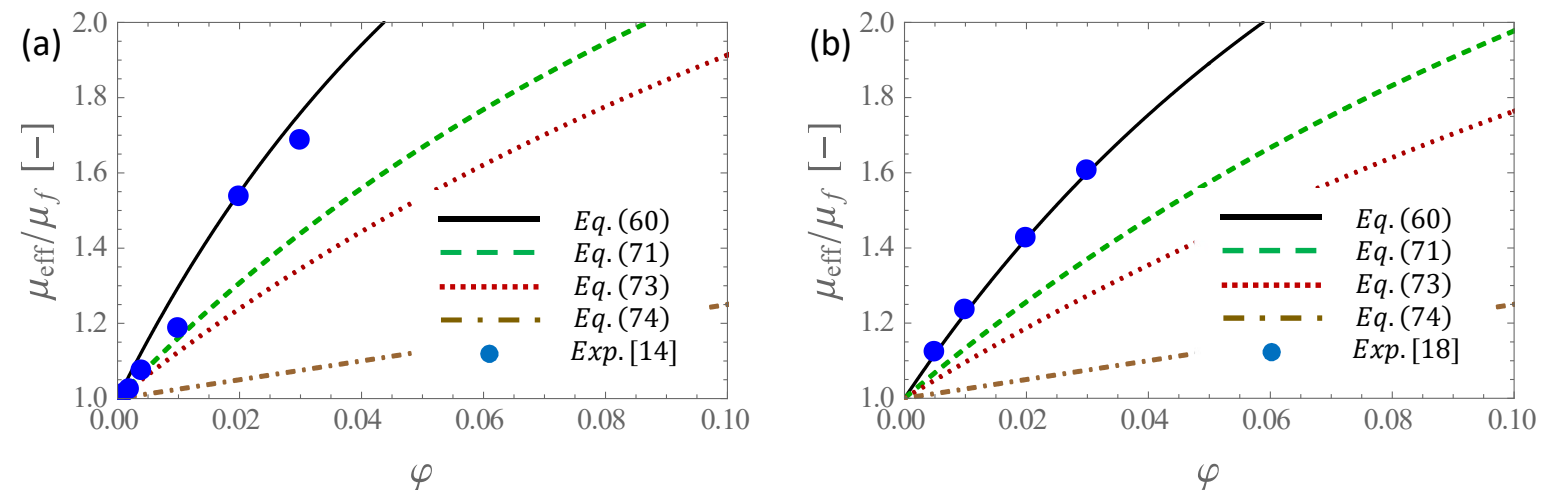

Figure 5: Relative effective viscosity of (a) $25 \mathrm{~nm} \mathrm{Fe} 3 \mathrm{O}_{4}$ nanoparticles and (b) $25 \mathrm{~nm} \mathrm{Al}_{2} \mathrm{O}_{3}$ nanoparticles, both dispersed in water.

The only difference in Fig. 5 is the material of the nanoparticle. This difference is in our model represented by two aspects: the density of the material and its interaction with the base fluid. As the difference of $\ell_{i}$ for the two nanodispersions is verified to be negligible with respect to the effect of the density, it can be put forward that the density of a nanoparticle, or rather its ratio with respect to that of the nanodispersion as the model shows, plays an important role. This is also responsible for the higher effective viscosity of the magnetite nanodispersion with respect to the alumina one. Our model suggests that a higher nanoparticle density results into a higher particle-fluid velocity difference (Eq. (34)), which means a higher drag force between the particles and the fluid, leading to a higher contribution to the effective viscosity of the nanodispersion. The effect of the particle density on the effective viscosity of a nanodispersion is an important observation, which is not explained before by a physics-based model. The effect of the liquid nanolayer and the density on the effective viscosity is furthermore shown by three carbon-based nanodispersions in Fig. 6. 

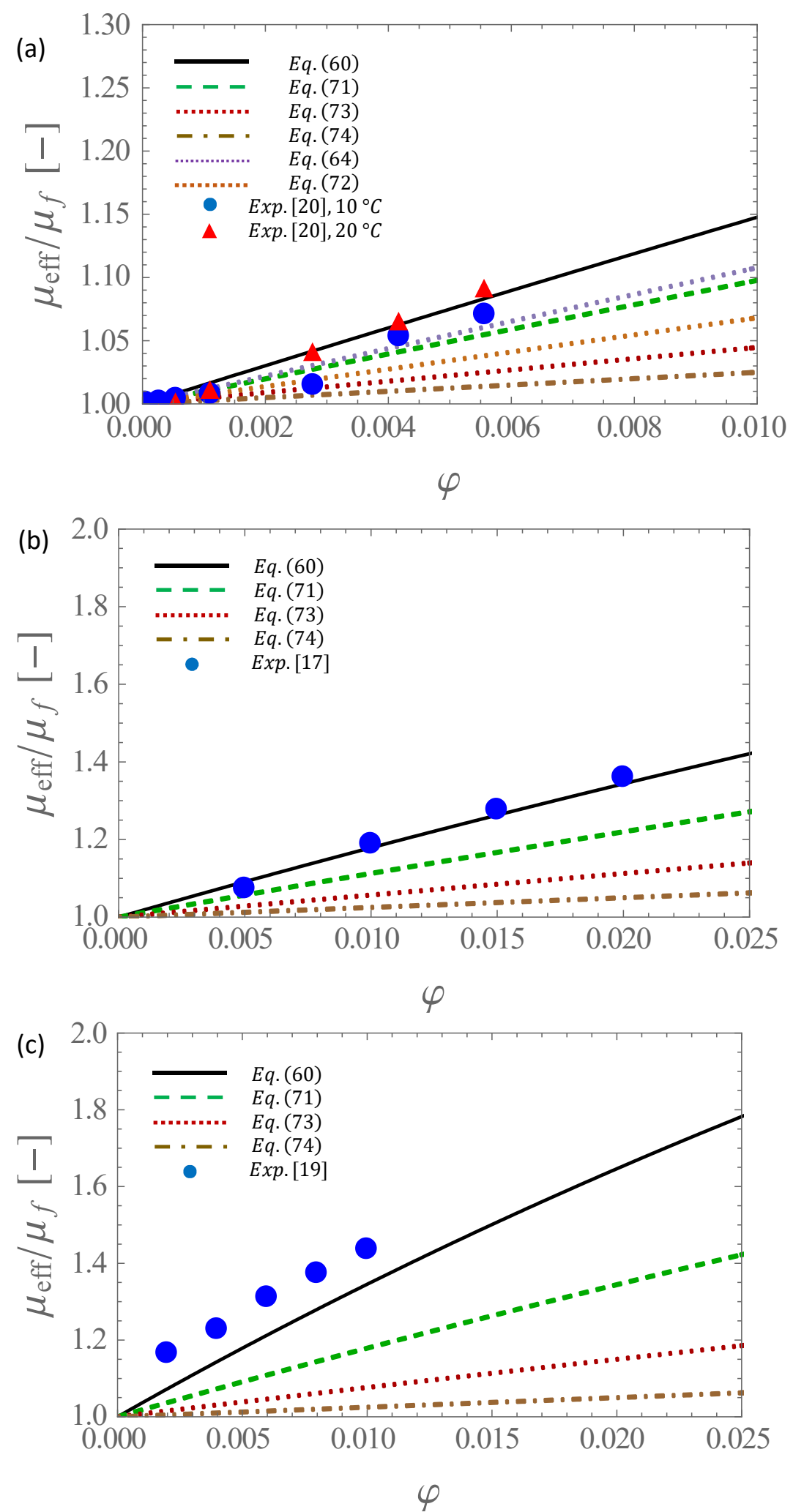

Figure 6: Relative effective viscosity of (a) $9 \mathrm{~nm}$ carbon nanotubes, (b) $8 \mathrm{~nm}$ graphite nanoparticles and (c) $10 \mathrm{~nm}$ diamond nanoparticles, all dispersed in water.

Before commenting these results, we make two remarks. First, from Fig. 6a we can notice that our model for spherical particles and for cylindrical particles agree rather well with one another so that we can keep the model for spherical particles for our discussion. Second, we used experimental data with approximately the same size for the nanoparticles in order to exclude 
the effect of size and focus more on the nanosized layer thickness and on the density. We can see that, although the nanolayer thickness for the graphite nanodispersion is lower than that for the CNT nanodispersion, the higher density of the former is enough to result into a considerably higher effective viscosity. This confirms that, although the effect of the nanolayer thickness is well-known, the density seems to have a far more important effect. It should be mentioned that most probably for nanoparticles of a size around $1 \mathrm{~nm}$ or less, the effect of the nanolayer thickness might well become more important, but these are dimensions that are out of the scope of a continuum model. For the nanodiamond dispersion the effective viscosity is clearly the hightest, which is not surprising noticing the higher nanolayer thickness and density of the diamond nanoparticles. This interplay between the interfacial energy (responsible for the formation of the interfacial nanolayer) and the density influencing viscous dissipation is represented in Fig. 7.

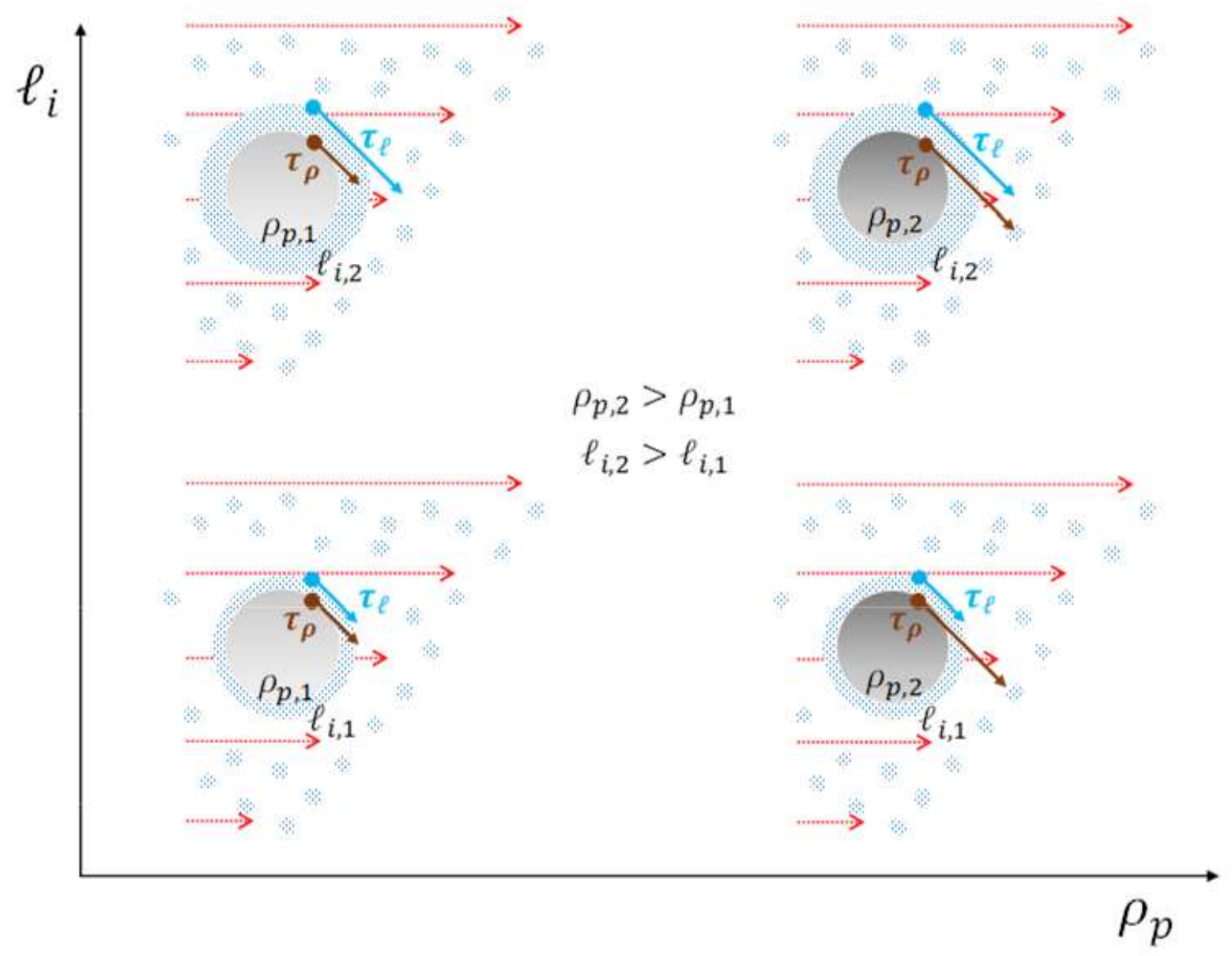

Figure 7: Schematic representation of viscous dissipation due to the interfacial surface energy $\tau_{\ell}$ and density $\tau_{\rho}$ for a given fluid density $\rho_{f}$, particle volume fraction $\varphi$ and temperature $T$.

As a final comparison, Fig. 8 presents a case where the nanoparticle is a mix between inorganic and carbon material and the base fluid is a mix between water and ethylene glycol. This result also shows a satisfactory agreement with the experimental data, which reinforces the physical mechanisms that are incorporated into our model in order to predict the effective viscosity of nanodispersions. 


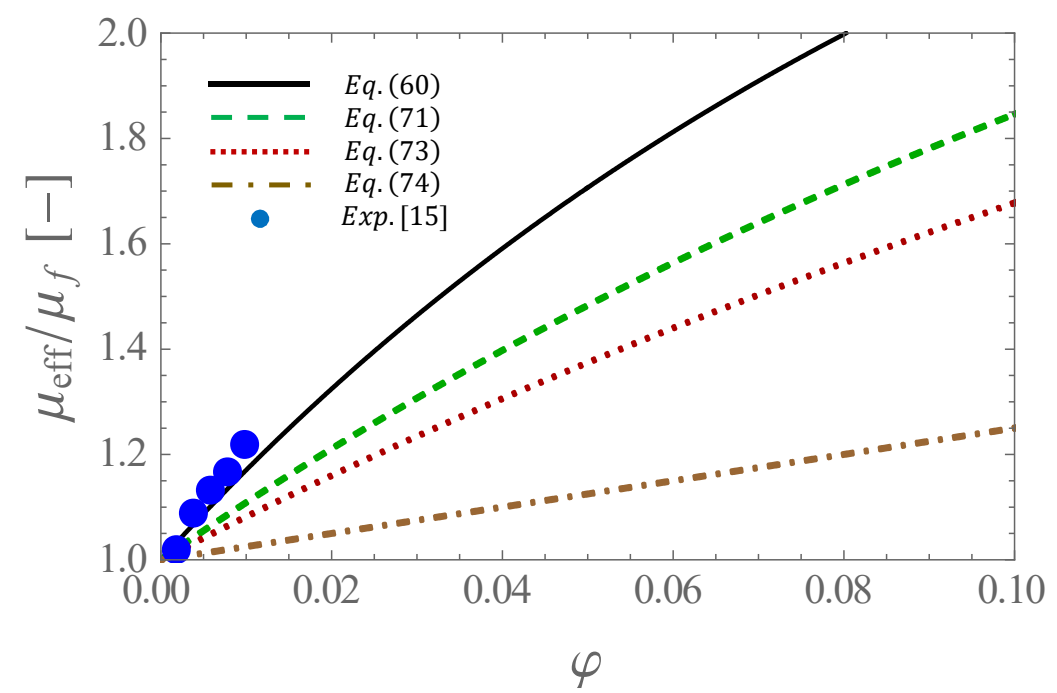

Figure 8: Relative effective viscosity of $30 \mathrm{~nm} \mathrm{SiC} \mathrm{nanoparticles} \mathrm{dispersed} \mathrm{in} 40 \mathrm{vol} \%$ ethyleneglycol and $60 \mathrm{vol} \%$ water.

\subsection{Whether there is a generality in the interfacial nanolayer thickness}

In an effort to propose a simplified universal expression, finding a value for the nanolayer thickness could be quite a difficult task. The most suitable would be to measure it experimentally as a material property. This is, however, not so straightforward and it might be argued to be empirical of character, which we would like to avoid in this paper. Alternatively, the nanolayer thickness could be calculated from molecular dynamics via interaction energies as discussed before. For many cases, it would, however, be useful to find out whether there is a general value that could be used if the correct value is unavailable. As mentioned before, the theoretically proposed nanolayer thickness is around $\ell_{i}=1-1.5 \mathrm{~nm}[50,51]$. It is therefore, interesting to assess the influence of the nanolayer thickness $\ell_{i}=1-1.5 \mathrm{~nm}$ on the effective viscosity for the studied cases. For interest, the case $\ell_{i}=0 \mathrm{~nm}$ is also considered. Fig. 4.5 shows the relative effective viscosity $\mu_{n f} / \mu_{f}$ as a function of the volume fraction for the various nanoparticle dispersions considered in the previous section. Here, for all cases, next to the liquid layer thickness tabulated in Table 1, also the theoretically proposed nanolayer thicknesses of $\ell_{i}=0,1$ and $1.5 \mathrm{~nm}$ are considered. Eqs. (60) and (64) are used and compared to the experimental data from Figs. 5-6 and 8.
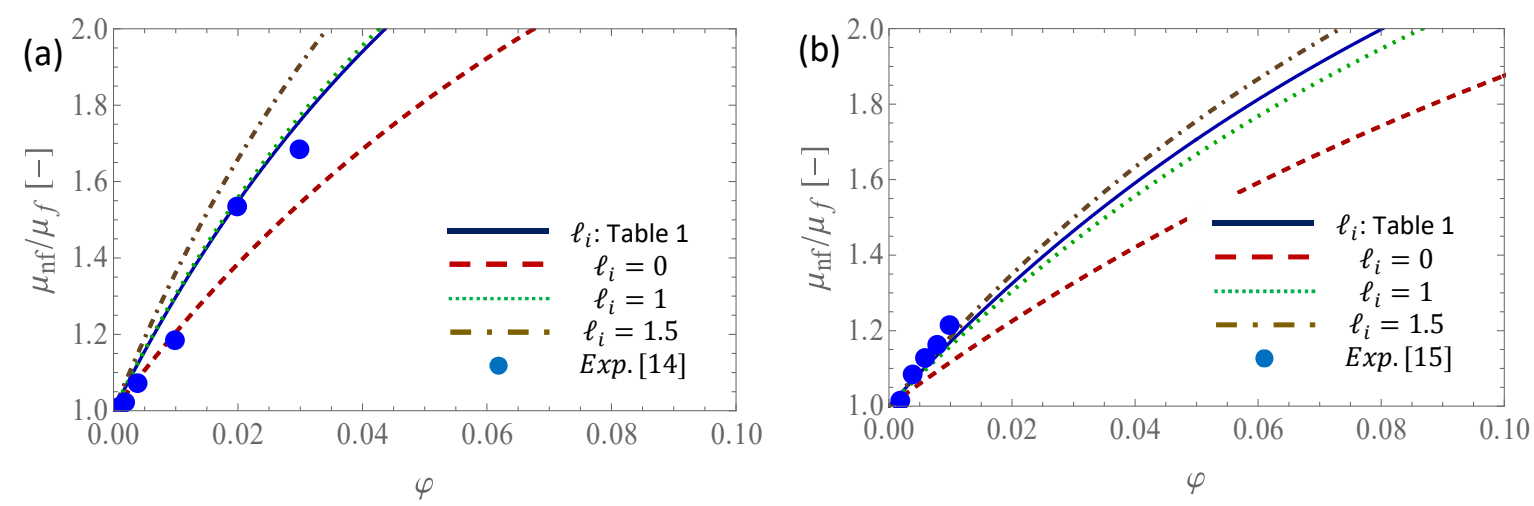

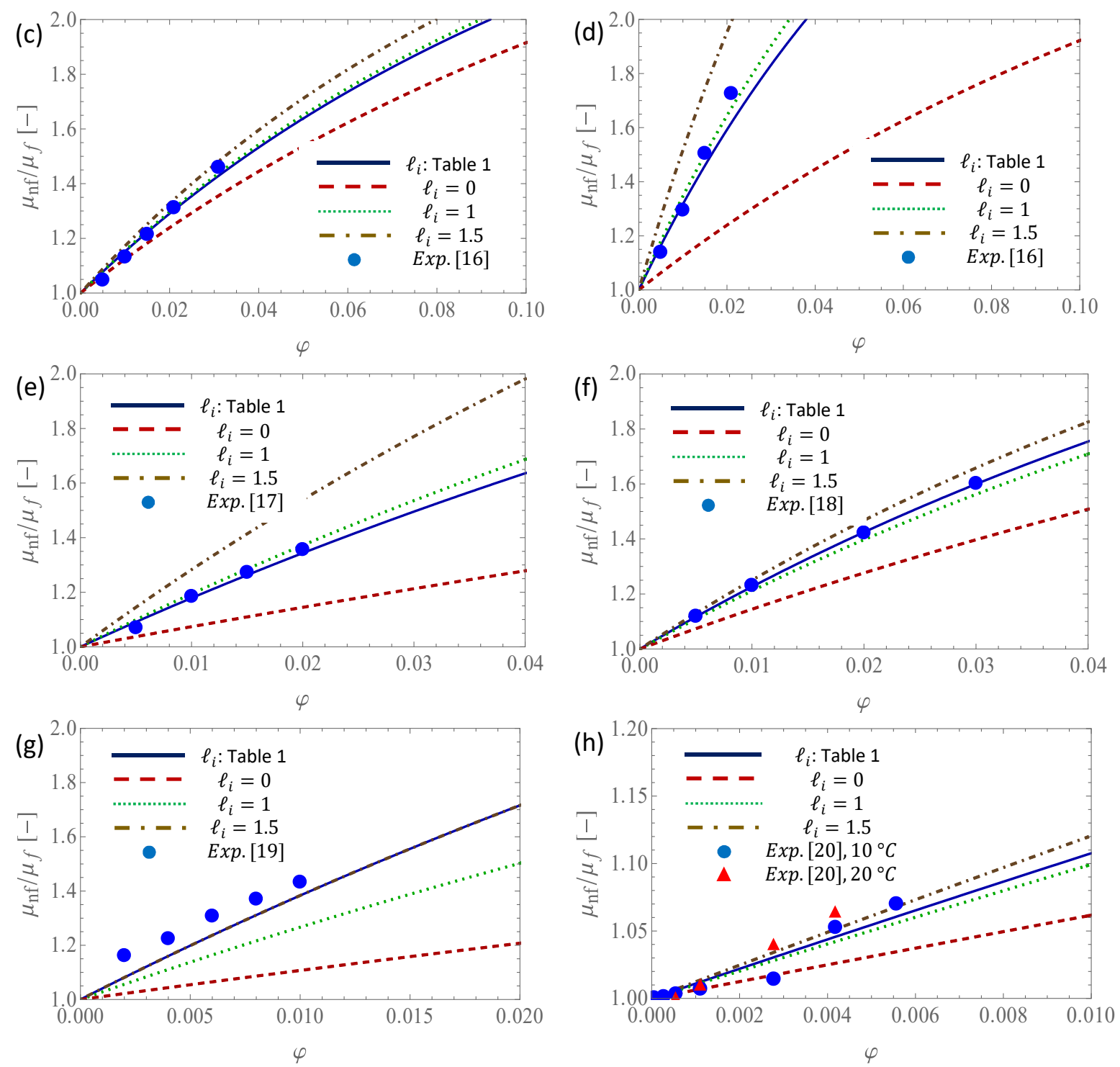

Figure 9: The relative effective viscosity $\mu_{n f} / \mu_{f}$ as a function of the volume fraction compared to experimental data: (a) $25 \mathrm{~nm} \mathrm{Fe} \mathrm{O}_{4}$ nanoparticles dispersed in water, (b) $30 \mathrm{~nm} \mathrm{SiC}$ nanoparticles dispersed in $40 \mathrm{vol} \%$ ethylene-glycol and $60 \mathrm{vol} \%$ water, (c) $43 \mathrm{~nm} \mathrm{Al} \mathrm{Al}_{3}$ nanoparticles dispersed in ethylene glycol, (d) $8 \mathrm{~nm} \mathrm{Al}_{2} \mathrm{O}_{3}$ nanoparticles dispersed in ethylene glycol, (e) $8 \mathrm{~nm}$ graphite nanoparticles dispersed in water, (f) $25 \mathrm{~nm} \mathrm{Al} \mathrm{O}_{3}$ nanoparticles dispersed in water, (g) $10 \mathrm{~nm}$ diamond nanoparticles dispersed in water, (h) $9 \mathrm{~nm}$ carbon nanotubes dispersed in water. The theoretically proposed liquid layer thicknesses of $\ell_{i}=0,1$ and $1.5 \mathrm{~nm}$ are also considered.

From the parametric series $\ell_{i}=0,1$ and $1.5 \mathrm{~nm}$, it is interesting to notice that for $\ell_{i}=O(1-$ 1.5) $\mathrm{nm}$ the model predicted the experimental values well, where it is also shown that the effect of the nanolayer thickness between values of 1 and $1.5 \mathrm{~nm}$ is relatively small. Although the effect is non-negligible for nanoparticle sizes below $10 \mathrm{~nm}$, it remains reasonable to state that it is rather correct to follow the theoretical prediction $[50,51]$ of nanolayer thicknesses of the order $\ell_{i}=O(1-1.5) \mathrm{nm}$. In choosing, for generality purposes, one value, it appeared that a nanolayer thickness around $1 \mathrm{~nm}$ seems to be the most appropriate one. 


\subsection{Universal expression for the viscosity of nanodispersions}

It appears from the results that neglecting the flux effect (neglecting the term $v_{f}(r)$ in (47)) still underestimates the viscosity of the nanodispersion. In order to take this effect into account, yet pursuing a universal expression for the viscosity keeping it as simple as possible, we propose to seek an approximated conversion of the $v_{f}$ term into the double derivative term $\frac{1}{r} \frac{\partial}{\partial r}\left(r \frac{\partial v_{f}}{\partial r}\right)$. For this purpose, we assume an ideal flow that can be both described by a local Darcy-flow (as discussed earlier) and a Poiseuille flow. The former would lead to $v_{f}=-\frac{R^{2}}{8} \frac{1}{\mu_{f}} \frac{\partial p}{\partial x}$ and the latter to $\frac{1}{r} \frac{\partial}{\partial r}\left(r \frac{\partial v_{f}}{\partial r}\right)=\frac{1}{\mu_{f}} \frac{\partial p}{\partial x}$. Comparison gives that

$v_{f} \approx-\frac{R^{2}}{8} \frac{1}{r} \frac{\partial}{\partial r}\left(r \frac{\partial v_{f}}{\partial r}\right)$

Filling this in in Eq. (47), using the definitions of $R$, neglecting the term $(1-\varphi)$ except there where it comes as $\varphi(1-\varphi)$, for the spherical and cylindrical particles, leads respectively, rearranging the terms, to

$$
\begin{aligned}
& \mu_{f}\left(1+\frac{9}{2} K n_{p, s}^{2}\left(c+\Xi_{s}\right)^{2}+\left(\frac{5}{2}+\frac{1}{4} \frac{\rho_{p}}{\rho}\left(1+\xi_{p f, s} \frac{\rho_{f}}{\rho}\right)\right)\left(c+\Xi_{s}\right)\right) \frac{1}{r} \frac{\partial}{\partial r}\left(r \frac{\partial v_{f}}{\partial r}\right)=\frac{\partial p}{\partial x}, \\
& \mu_{f}\left(1+\frac{9}{2} K n_{p, c}^{2}\left(c+\Xi_{c}\right)^{2}+\left(\frac{5}{2}+\frac{1}{4 \frac{\pi}{2+\pi}} \frac{\rho_{p}}{\rho}\left(1+\xi_{p f, c} \frac{\rho_{f}}{\rho}\right)\right)\left(c+\Xi_{c}\right)\right) \frac{1}{r} \frac{\partial}{\partial r}\left(r \frac{\partial v_{f}}{\partial r}\right)=\frac{\partial p}{\partial x}
\end{aligned}
$$

with $\xi_{p f, s}$ and $\xi_{p f, c}$ are given, respectively, by Eqs. (25) and (52). For nanodispersions where the base fluid is a liquid, we assume that $K n_{p, s}^{2} \ll 1$ so that the corresponding term could be neglected. Using expressions (25), (52), (62) and (66), the effective viscosities for spherical and cylindrical nanodispersions are given, respectively, by

$$
\begin{aligned}
& \mu_{n f}=\mu_{f}\left(1+\left(\frac{5}{2}+\frac{1}{4} \frac{\rho_{p}}{\rho}\left(1+\xi_{p f, s} \frac{\rho_{f}}{\rho}\right)\right)\left(c+\xi_{p f, s} c(1-c)\right)\right) \\
& \mu_{n f}=\mu_{f}\left(1+\left(\frac{5}{2}+\frac{1}{4 \frac{\pi}{2+\pi}} \frac{\rho_{p}}{\rho}\left(1+\xi_{p f, c} \frac{\rho_{f}}{\rho}\right)\right)\left(c+\xi_{p f, c} c(1-c)\right)\right)
\end{aligned}
$$

recalling that $\xi_{p f, s}=2\left(\left(1+\frac{\ell_{i}}{a_{p, s}}\right)^{2}-1\right), \xi_{p f, c}=2 \frac{\ell_{i}}{a_{p, c}}$ and $c=\frac{\varphi \rho_{p}}{\rho}$. The approximate models (78) and (79) are compared with the complete model, using the experimental data from Table 1 and the results are presented in Fig. 10. 

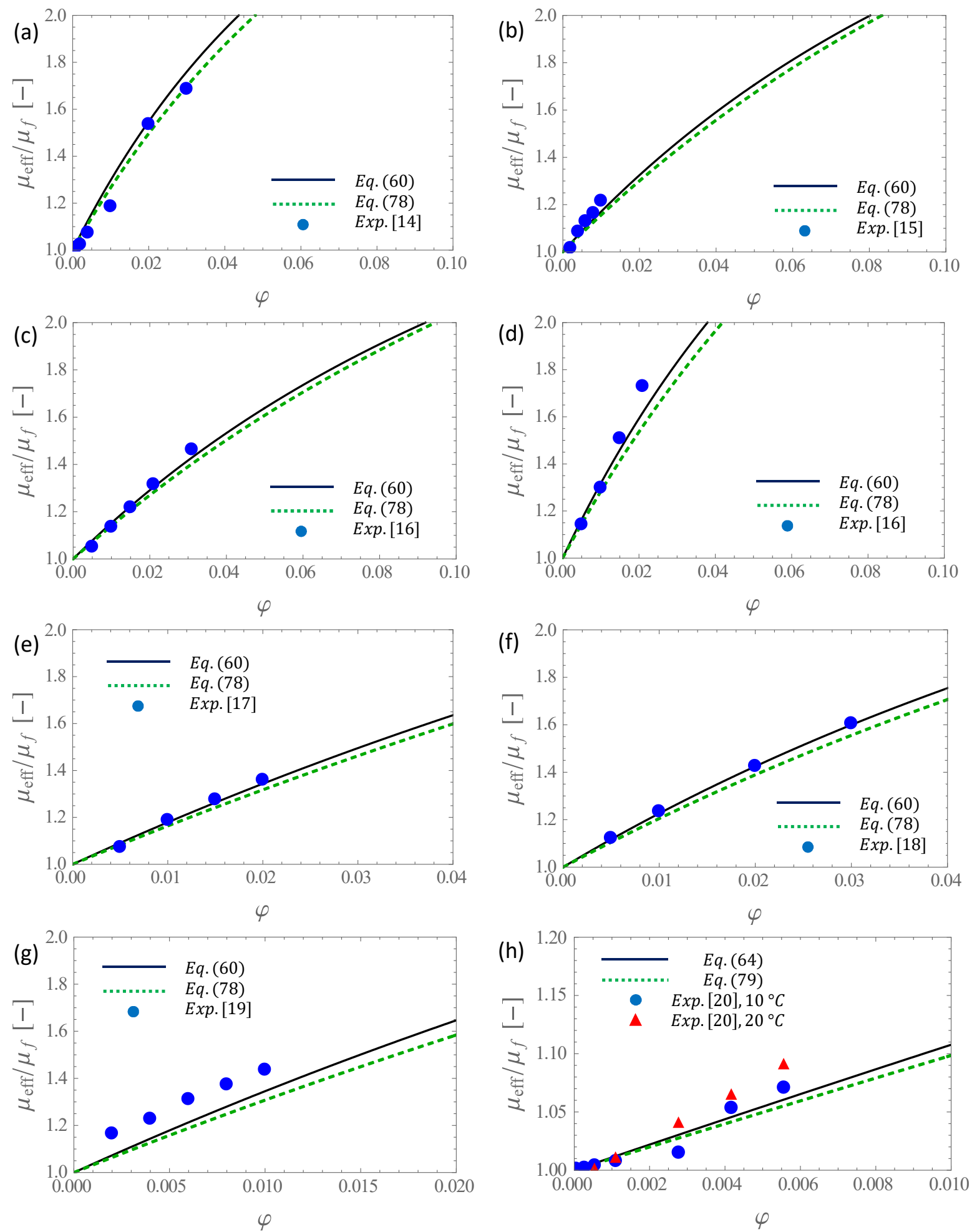

Figure 10: Relative effective viscosity given by the full model (60)-(67) and the approximate one (78)-(79), compared to the experimental data as in Fig. 9.

Fig. 10 shows that the approximate model represents quite well the experimental data and is very close to the full model. In order to test the universality of the approximate model, with respect to the effective viscosity of nanodispersions, it should be compared to other experimental data with different sets of parameters (density, type of material, size). As is discussed earlier, we argued that it would be reasonable to take $\ell_{i}=1.0 \mathrm{~nm}$. As such, the 
proposed universal expression of the effective viscosity of nanodispersions is, apart from the fluid/matrix density, solely dependent on the particle's size, volume fraction and density.

\subsection{Application to solid-liquid and polymeric nanodispersions and emulsions}

We use our universal expression to compare with other experimental data concerning ceramic, carbon and polymer solid-liquid nanodispersions [71-74], polymeric nanodispersions [75,76] and stabilized liquid-liquid nano/micro dispersions (emulsions) [77-80]. It is important to note that it concerns here stabilized emulsions, where the nano/micro-droplets can be considered as hard spheres. The material properties are given in Table 2. Properties that are not standardly available (from the reference or from standard handbooks, such as [81]), are commented below the table. In Table 2, W stands for water, EG for ethylene glycol, SWCNT for single-wall carbon nanotubes, PSL for polystyrene latex, PEG400 and PEO400 for, respectively, polyethylene glycol and poly-ethylene oxide (where 400 stands for the molar mass in $\mathrm{g} / \mathrm{mol}$ ) and GTO for glyceryl tri-octanoate.

Table 2: Material properties for other nanodispersions and stabilized emulsions

\begin{tabular}{|c|c|c|c|c|c|}
\hline Nanofluids & $\begin{array}{l}T_{\text {ref }} \\
{\left[{ }^{\circ} \mathrm{C}\right]}\end{array}$ & $\rho_{f}\left[\mathrm{~kg} / \mathrm{m}^{3}\right]$ & $\begin{array}{l}\rho_{p} \\
{\left[\mathrm{~kg} / \mathrm{m}^{3}\right]}\end{array}$ & $\begin{array}{l}2 a_{p} \\
{[\mathrm{~nm}]}\end{array}$ & Ref. \\
\hline $\mathrm{CuO}-\mathrm{W}$ & 25 & 997 & 6310 & 11 & [71] \\
\hline SWCNT - EG & 60 & 1116 & 1600 & 45 & [72] \\
\hline $\mathrm{CuO}-$ Coconut oil $^{\mathrm{a}}$ & 55 & $897^{\mathrm{b}}$ & 6310 & 40 & [73] \\
\hline $\mathrm{PSL}-\mathrm{W}$ & 20 & 998 & 1055 & 40 & [74] \\
\hline $\mathrm{SiO}_{2}-\mathrm{PEG} 400$ & 25 & 1130 & $1600^{c}$ & 127 & [75] \\
\hline $\mathrm{SiO}_{2}-\mathrm{PEO} 400$ & 75 & $1170^{\mathrm{d}}$ & $1600^{c}$ & 44 & [76] \\
\hline Silicon oil - W & 25 & 997 & 971 & $\begin{array}{ll}220 & \& \\
550 & \\
\end{array}$ & {$[77]$} \\
\hline White mineral oil $-\mathrm{W}$ & 25 & 997 & 850 & 14000 & [78] \\
\hline GTO - W & 25 & 997 & 956 & 110 & [79] \\
\hline W - Mineral oil & 10 & 870 & 1000 & 30000 & [80] \\
\hline
\end{tabular}

${ }^{a}$ Values at shear rates of 3.67, 7.34 and $14.68 \mathrm{~s}^{-1}[73]$

${ }^{\mathrm{b}}$ From [82]

${ }^{c}$ Value deviates from standard one, given by $[75,76]$

${ }^{\mathrm{d}}$ Value estimated from [83] and corrected for the temperature by extrapolation following the behaviour of PEG400 [84]
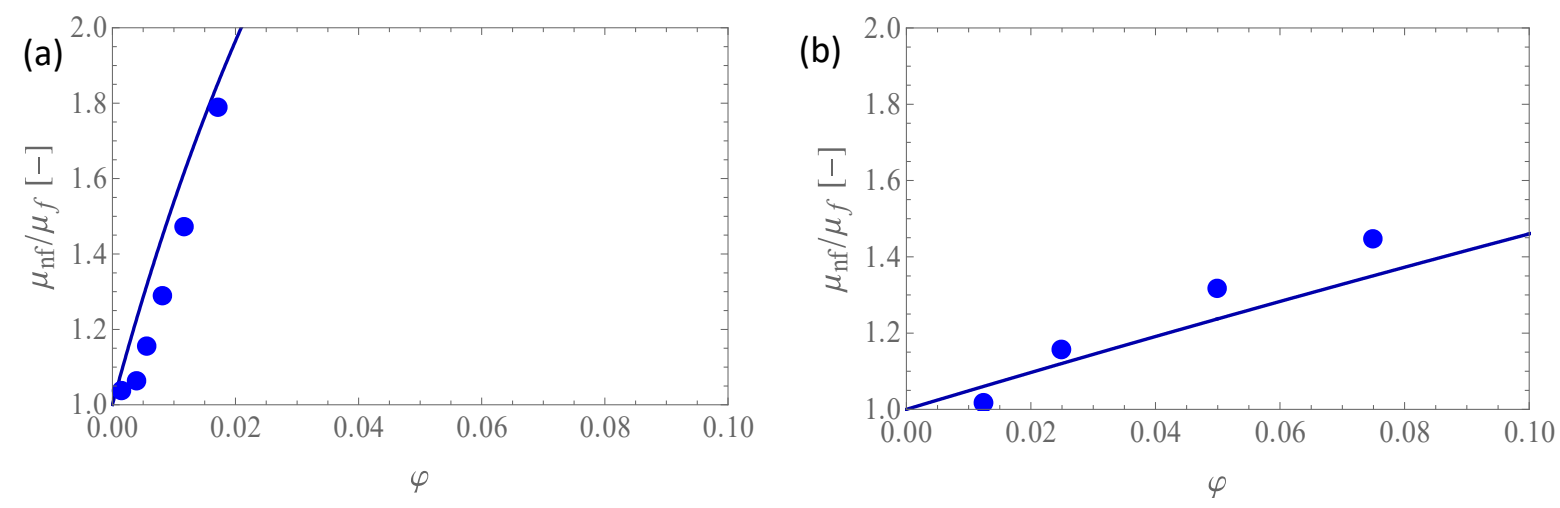

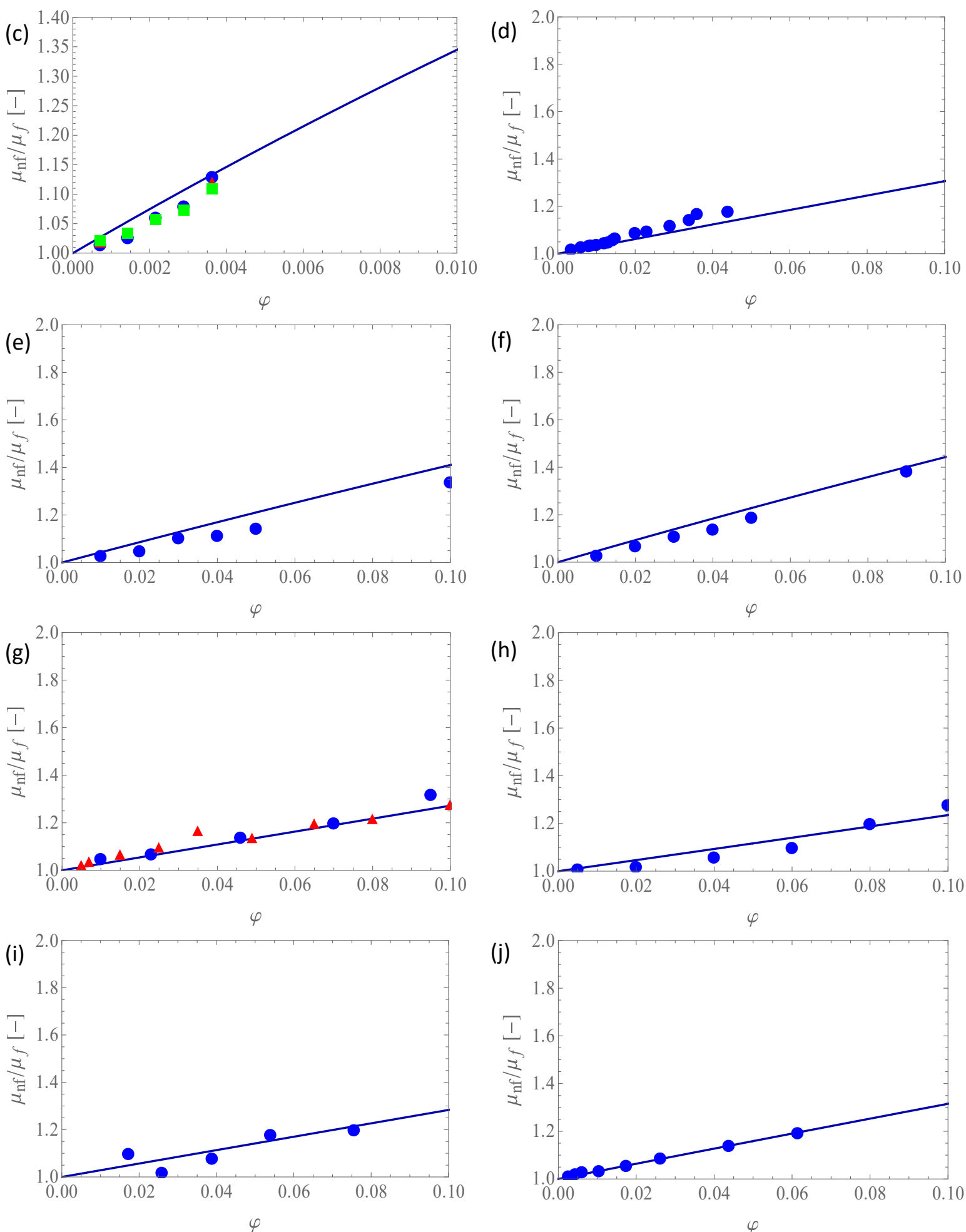

Figure 11: The relative effective viscosity as a function of the volume fraction from expressions (78) for spherical fillers and (79) for cylindrical ones. The solid lines represent the model and the markers the experimental data, which correspond to: (a) $11 \mathrm{~nm} \mathrm{CuO}$ nanoparticles in water, (b) $45 \mathrm{~nm}$ single-walled carbon nanotubes in ethylene glycol, (c) $40 \mathrm{~nm} \mathrm{CuO}$ nanoparticles in coconut oil for three low-shear rates in Newtonian regime, (d) $40 \mathrm{~nm}$ polystyrene latex nanoparticles in water, (e) $127 \mathrm{~nm} \mathrm{SiO} 2$ nanoparticles in poly-ethylene glycol, (f) $44 \mathrm{~nm} \mathrm{SiO}_{2}$ nanoparticles in poly-ethylene oxide, (g) 220 and $550 \mathrm{~nm}$ silicon oil nanodroplets in water, (h) $14 \mu \mathrm{m}$ white mineral oil microdroplets in water, (i) $110 \mathrm{~nm}$ glyceryl tri-octanoate nanodroplets in water, (j) $30 \mu \mathrm{m}$ water microdroplets in mineral oil. 
Fig. 11 shows that the approximate model agrees well with several experimental data, suggesting its universality. Furthermore, besides the well-known size and nanolayer effects, it emphasizes the clear universal relation between the density of the nanoparticles or nano/micro droplets in emulsion on the effective viscosity. One could then argue that the density not only plays an important role, but could, in many case, well be the most important one at constant temperature. The densities alone, could even be sufficient, using Eqs. (78)-(79), to predict the effective viscosity of nanodispersions as a function of their content in nanoparticles or droplet emulsions.

\section{Conclusions}

Nanodispersions are considered in this paper to be, from a fluid-flow point of view, a heterogeneous system, where the stabilized particles are dispersed in a base fluid. The base fluid considered is a liquid, whereas the particle could be a solid spherical nanoparticle, a nanotube or even a stabilized liquid droplet, the latter of which constitutes an emulsion. Such systems find many applications and one of the main properties to control is their rheology. Especially the viscosity appears to behave differently from one nanodispersion to another and a universal expression predicting its behaviour is necessary. In striving to attain such a relation, many mechanisms that come into play should be well represented, retaining altogether a digestable formulation. This work proposes a framework, based on extended thermodynamics and fluid dynamics, that incorporates the effects of size, particle kinetics, mass density, interfacial nanolayer and volume fraction on the effective viscosity of nanodispersions. The model begins by proposing an evolution equation for the fluid velocity, allowing for a particlefluid flux and viscous dissipation due to the presence of nanoparticles and particle kinetics. By making an anology to a porous system, where the fluid flows in between the stabilized nanoparticles, solving the evolution equation and comparing to a developed stationary flow profile lead to resuming all the considered effects in the evoluation equation into one effective property defined as the effective viscosity. This equation was compared satisfactorily to several experimental data for nanodispersions with spherical and cylindrical nanodispersions. Asymptotic models allowed appreciating the importance of the included mechanisms. A much simpler relation is then proposed, Eqs. (78) and (79) and equivalently validated against a great number of ceramic and polymeric nanodispersions, as well as nano/micro emulsions, suggesting its universality for stable nanodispersions. It appeared that for small nanoparticles, the interfacial nanolayer influenced in an enhancing way greatly the effective viscosity. The nanolayer's influence is modelled as a competition between the Brownian surface energy and the net attractive interchange energy. As the nanoparticles size is smaller, for a given volume fraction, the total interfacial surface becomes larger, leading to a higher contribution to viscous dissipation. It also appeared that the flux term (kinetic contribution of the particle-fluid interaction) is not to be neglected and makes clearly part of one of the mechanisms of viscous flow. Last but certainly not least, the density of the nanoparticles with respect to that of the dispersion seemed to have the most important influence on the effective viscosity, explained as viscous dissipation due to a higher drag force caused by a higher particle-fluid velocity for higher nanoparticle densities.

In contrast with several other papers on the subject [21-28], wherein ad hoc relations involving adjustable parameters are proposed, we have tried to provide a model with strong theoretical bases and to back most of the assumptions underlying the study by physically grounded justifications, without adding any empirical coefficients, but merely using tabulated and 
measured material properties. Comparison with experimental data exhibits a good agreement, attesting of the quality and soundness of the theoretical model. For simplicity, the analysis has been limited to one-dimensional and linearized situations, whereas temperature effects have been omitted. There is, of course, room for further extensions of the present model like considering metastable dispersions of hydrophobic nanoparticles, considering concentration nanodispersion with inter-particle interaction or introducing rheological properties, such as the dependence of the material coefficients on imposed shear rates. Nonetheless, the universal expression, proposed in this work, has shown that there is a universal relation between the effective viscosity of nanodispersions and their densities.

\section{Acknowledgements}

H.M. acknowledges financial support from BelSPo.

Appendix: extended entropy relation

Following the line of thought of EIT, whose main idea is to elevate the dissipative fluxes, like the heat or/and the mass fluxes to the status of independent variable, $\boldsymbol{J}_{f}$ is an independent state variable. For pedagogical purpose, let us study the simple problem of matter diffusion in a twocomponent mixture of mass fractions $c_{1}$ and $c_{2}$, the temperature $T$ is assumed to be uniform. The main idea underlying EIT is to elevate the thermodynamic fluxes, here the diffusion fluxes $\boldsymbol{J}_{1}$ and $\boldsymbol{J}_{2}$ to the status of independent variables, at the same level as the classical concentration varaiables. According to the definition of the barycentric velocity, it is directly seen that $\boldsymbol{J}_{1}+$ $J_{2}=0$. Moreover, since $c_{1}+c_{2}=1$, it follows that the set of independent variables is given by $c_{1}$ and $J_{1}$. Assuming that the entropy $s$ per unit mass of the system depends on both kinds of variables, one has $s=s\left(c_{1}, \boldsymbol{J}_{1}\right)$ or, in terms of the material time derivative

$\frac{d s}{d t}=\frac{\partial s}{\partial c_{1}} \frac{d c_{1}}{d t}+\frac{\partial s}{\partial J_{1}} \cdot \frac{d J_{1}}{d t}=-\frac{\eta}{T} \frac{d c_{1}}{d t}-\alpha J_{1} \cdot \frac{d J_{1}}{d t}$,

wherein use has been made of the classical definition $\frac{\eta}{T}=-\frac{\partial s}{\partial c_{1}}$, with $\eta$ designating the difference $\eta_{1}-\eta_{2}$ between the chemical potentials of both constituents and wherein it has been assumed that $\frac{\partial s}{\partial J_{1}}$ is a linear function of $\boldsymbol{J}_{1}$ with $\alpha$ a phenomenological coefficient to be positive to guarantee that $s$ is maximum at equilibrium (Jou et al 2010). Entropy is also assumed to obey a time evolution equation of the general form

$\rho \frac{d s}{d t}=-\nabla \cdot \boldsymbol{J}^{s}+\sigma^{s}, \quad$ with $\sigma^{s} \geq 0$

$\sigma^{S}$ is the rate of entropy production imposed to be positive definite in virtue of the second law of themodynamics and $\boldsymbol{J}^{S}$ is the entropy flux classically given by

$\boldsymbol{J}^{S}=-\frac{\eta}{T} \boldsymbol{J}_{1}$

This result (A3) is easily obtained by setting $\alpha=0$ in (A1) and substituting $\frac{d c_{1}}{d t}$ by the mass conservation law

$\rho \frac{d c_{1}}{d t}=-\nabla \cdot \boldsymbol{J}_{1}$ 
By comparison with the time evolution (A1) of $s$, it is then directly checked that expression of $\boldsymbol{J}^{s}$ is given by (A3). Filling (A3) and (A1), with the use of (A4) and $\alpha=0$, into (A2), we obtain the classical expression for the entropy production

$\sigma^{s}=-J_{1} \cdot \frac{\nabla \eta}{T} \geq 0$

However, in presence of non-localities which are especially relevant in micro and nanosystems, it is rather natural to admit that $\boldsymbol{J}^{S}$ depends, in addition, on the gradients of the diffusion flux $J_{1}$, for example,

$\boldsymbol{J}^{s}=-\frac{\eta}{T} \boldsymbol{J}_{1}+\gamma \boldsymbol{J}_{1} \cdot \nabla \boldsymbol{J}_{1}$,

wherein $\gamma$ is a coefficient to be determined later on. The final task consists in deriving the time evolution equation of the state variables. The one corresponding to the classical mass fraction variable is given by (A4), while the time evolution equation of the diffusion flux is obtained by substituting (A1) and (A6) in (A2). The corresponding entropy production is now given by

$\sigma^{S}=\boldsymbol{J}_{1} \cdot\left(-\frac{\nabla \eta}{T}-\rho \alpha \frac{d \boldsymbol{J}_{1}}{d t}+\gamma \nabla^{2} \boldsymbol{J}_{1}\right)+\gamma \nabla \boldsymbol{J}_{1} \otimes \nabla \boldsymbol{J}_{1} \geq 0$

with $\otimes$ standing for the tensorial product.

\section{References}

[1] H. Machrafi, An extended thermodynamic model for size-dependent thermoelectric properties at nanometric scales: Application to nanofilms, nanocomposites and thin nanocomposite films, Appl. Math. Mod. (2016), 40, 2143-2160.

[2] F. Yu, Y. Chen, X. Liang, J. Xu, C. Lee, Q. Liang, P. Tao, T. Deng, Dispersion stability of thermal nanofluids, Progress in Natural Science: Materials International 27 (2017) 531-542.

[3] H. Machrafi, Enhancement of a photovoltaic cell performance by a coupled cooled nanocomposite thermoelectric hybrid system, using extended thermodynamics, Current Appl. Phys. (2017), 17, 890-911.

[4] J. Taha-Tijerina, T.N. Narayanan, G. Gao, M. Rohde, D.A. Tsentalovich, M. Pasquali, P.M. Ajayan, Electrically Insulating Thermal Nano-Oils Using 2D Fillers, ACS Nano 6 (2012) 1214-1220.

[5] A. Cardellini, M. Fasano, M.B. Bigdeli, E. Chiavazzo, P. Asinari, Thermal transport phenomena in nanoparticle suspensions, J. Phys. Condens. Matter 28 (2016) 483003.

[6] Y. Lin, S. Sun, Q. Zhang, H. Shen, Q. Shao, L. Wang, W. Jiang, W. Jiang, Preparation of AgNPs/Ca3Co4O9 nanocomposites with enhanced thermoelectric performance, Materials Today Communications (2016), 6, 44-49.

[7] H. Machrafi, G. Lebon, C.S. Iorio, Effect of volume-fraction dependent agglomeration of nanoparticles on the thermal conductivity of nanocomposites: Applications to epoxy resins, filled by $\mathrm{SiO} 2, \mathrm{AlN}$ and $\mathrm{MgO}$ nanoparticles, Composites Science and Technology (2016), 130, 78-87.

[8] Z. Hai, M.K. Akbari, Z. Wei, C. Xue, H. Xu, J. Hu, L. Hyde, S. Zhuiykov, TiO2 nanoparticles-functionalized two-dimensional WO3 for high-performance supercapacitors developed by facile two-step ALD process, Materials Today Communications (2017), 12, 55-62.

[9] R. Ahmad, O.S. Wolfbeis, Y.B. Hahn, H.N. Alshareef, L. Torsi, K.N. Salama, Deposition of nanomaterials: A crucial step in biosensor fabrication, Materials Today Communications (2018), 17, 289-321.

[10] Y. Chevalier, M.A. Bolzinger, S. Briançon (2015) Pickering Emulsions for Controlled Drug Delivery to the Skin. In: Dragicevic N., Maibach H. (eds) Percutaneous Penetration Enhancers Chemical Methods in Penetration Enhancement. Springer, Berlin, Heidelberg.

[11] M. Kumar, R.S. Bishnoi, A.K. Shukla, C.P. Jain, Techniques for formulation of nanoemulsion drug delivery system: A Review, Prev. Nutr. Food Sci. 2019, 24, 225-234

[12] N.A.N. Azmi, A.A.M. Elgharbawy, S.R. Motlagh, N. Samsudin, H.M. Salleh, Nanoemulsions: factory for food, pharmaceutical and cosmetics (2019), Processes 7, 617.

[13] R. Saidur, K.Y. Leong, H. Mohammad, A review on applications and challenges of nanofluids, Renew. Sustain. Energy Rev. 15 (2011), 1646-1668. 
[14] D. Toghraie, S. M. Alempour, M. Afrand, Experimental determination of viscosity of water based magnetite nanofluid for application in heating and cooling systems, Journal of Magnetism and Magnetic Materials (2016) 417, 243-248.

[15] X. Li, C. Zou, Thermo-physical properties of water and ethylene glycol mixture based SiC nanofluids: an experimental investigation, International Journal of Heat and Mass Transfer (2016) 101, 412-417.

[16] M.J. Pastoriza-Gallego, L. Lugo, J.L. Legido, M.M. Piñeiro, Thermal conductivity and viscosity measurements of ethylene-glycol-based $\mathrm{Al}_{2} \mathrm{O}_{3}$ nanofluids, Nanoscale Research Letters (2011) 6, 221.

[17] A.S. Dalkilic, A. Cebi, A. Celen, O. Yildiz, O. Acikgoz, C. Jumpholkul, M. Bayrak, K. Surana, S. Wongwises, Prediction of graphite nanofluids' dynamic viscosity by means of artificial neural networks, Int. Comm. Heat Mass Transf. 73 (2016) 33-42.

[18] J.B. Mena, A.A. Ubices de Moraes, Y.R. Benito, G. Ribatski, J.A.R. Parise, Extrapolation of $\mathrm{Al}_{2} \mathrm{O}_{3}$-water nanofluid viscosity for temperatures and volume concentrations beyond the range of validity of existing correlations, Appl. Therm. Eng. 51 (2013) 1092-1097.

[19] L.S. Sundar, M.J. Hortiguela, M.K. Singh, A.C.M. Sousa, Thermal conductivity and viscosity of water based nanodiamond (ND) nanofluids: An experimental study, Int. Comm. Heat Mass Trans. 76 (2016) 245-255.

[20] S. Halelfadl, P. Estellé, B. Aladag, N. Doner, T. Maré, Viscosity of carbon nanotubes water-based nanofluids: Influence of concentration and temperature, Int. J. Therm. Sc. 71 (2013) 111-117.

[21] A. Einstein, Eine Neue Bestimmung der Molekuldimensionen, Annals Phys. 324(2) (1906) 874-885.

[22] M. Mooney, The viscosity of concentrated suspensions of rigid spheres, J. colloid.Sci 6(2) (1951)162-170.

[23] I.M. Krieger, J.D. Thomas, A mechanism for non-Newtonian flow in suspensions of spherical particles, Trans. Soc. Rheol. 3(1) (1957) 137-152.

[24] 1.E. Nielsen, Generalized equation for the elastic moduli of composite materials, J Appl. Phys. 41(11) (1970) 4626-4627.

[25] G.K. Batchelor, The effect of Brownian motion on the bulk stress in a suspension of spherical particles, J. Fluid Mech. 83 (1977) 137-152.

[26] C. Brinkman, The viscosity of concentrated suspensions and solutions, J. Chem. Phys. 20(4) (1952) 571.

[27] X. Wang, X.Xu, S.U.S. Choi, Thermal conductivity of nanoparticle fluid mixture, J. Thermophys. Heat Transfer 13 (1999) 474-480.

[28] N. Masoumi, N. Shrabi, A.Behzadmehr, A new model for calculating the effective viscosity of nanofluids, J. Appl. Phys. 42 (2009) 055501.

[29] D. Jou, J. Casas-Vàzquez, G Lebon.: Extended Irreversible Thermodynamics. 4th edition, Springer, New York (2010).

[30] H. Machrafi, Extended Non-Equilibrium Thermodynamics: From Principles to Applications in Nanosystems. $1^{\text {st }}$ edition, Taylor \& Francis Group, London (2019).

[31] H. Machrafi , G. Lebon, General constitutive equations for heat transport at small length and high frequencies with extension to mass and electrical scales transport, Appl. Math. Lett 22 (2016) 30-37.

[32] H. Machrafi, G.Lebon, Fluid flow through porous and nanoporous media within the prism of extended thermodynamics: emphasis on the notion of permeability, Microfluidics and Nanofluidics, 22:65 (2018)1-12.

[33] G. Lebon, D. Jou, J. Casas-Vazquez, Understanding Non-equilibrium Thermodynamics, Springer, Berlin (2008).

[34] G. Lebon, D. Lhuillier, A. Palumbo, A thermodynamic description of thermo-diffusion in suspensions of rigid particles, Eur. Phys. J. Special Topics 146, 3-12 (2007).

[35] M. Grmela, G. Lebon, D. Lhuillier, A comparative study of the coupling of flow with non-Fickean thermodiffusion. Part II: GENERIC (2003), Journal of Non-Equilibrium Thermodynamics, 28, 23-50.

[36] H. Machrafi, On the chemical potential of nanoparticle dispersion, In Press https://doi.org/10.1016/j.physleta.2020.126485 (2020).

[37] K.R. Sharma, Nanostructuring of nanorobots for use in nanomedicine, International Journal of Engineering and Technology (2012), 2, 116-134.

[38] M.A. Van der Hoef, R. Beetstra, J.A.M. Kuipers (2005), Lattice-Boltzmann simulations of low-Reynoldsnumber flow past mono-and bidisperse arrays of spheres: results for the permeability and drag force, Journal of Fluid Mechanics 528, 233-254.

[39] L. Wang, J. Fan, Toward nanofluids of ultra-high thermal conductivity, Nanoscale Research Letters (2011), $6,153$.

[40] K. Voïtchovsky, J.J. Kuna, S.A. Contera, E. Tosatti, F. Stellacci, Direct mapping of the solid-liquid adhesion energy with subnanometre resolution, Nature Nanotechnology (2010), 5, 401-405. 
[41] K. Wu, Z. Chen, J. Li, X. Li, J. Xu, X. Dong, Wettability effect on nanoconfined water flow (2017), PNAS $114,13$.

[42] C.J. van Oss, Interfacial forces in aqueous media, Marcel Dekker, New York (1994).

[43] A.H. Nikoo, A. Kalantariasl, M.R. Malayeri, Propensity of gypsum precipitation using surface energy approach, Journal of Molecular Liquids (2020), 300, 112320.

[44] Yu C. J., Richter A. G., Datta A., Durbin M. K., Dutta P.: Molecular layering in a liquid on a solid substrate: an X-ray reflectivity study, Physica B 283, 27-31 (2000).

[45] P.A. Mante, C.C, Chen, Y.C. Wen, H.Y. Chen, S.C. Yang, Y.R. Huang, I.J. Chen, Y.W. Chen, V. Gusev, M.J. Chen, J.L. Kuo, J.K. Sheu, C.K. Sun, Probing hydrophilic interface of solid/liquid-water by nanoultrasonics, Scientific Reports 4 (2014) 6249.

[46] C. Gerardi, D. Cory, J. Buongiorno, L.W. Hu, T. McKrell, Nuclear magnetic resonance-based study of ordered layering on the surface of alumina nanoparticles in water, Applied Physics Letters 95 (2009) 253104.

[47] Xue L., Keblinski P., Phillpot S. R., Choi S. U. S., Eastman J. A.: Effect of liquid layering at the liquid-solid interface on thermal transport, Int. J. Heat Mass Transfer 47, 4277-4284 (2004).

[48] A. Cardellini, M. Fasano, E. Chiavazzo, P. Asinari, Interfacial water thickness at inorganic nanoconstructs and biomolecules: Size matters, Physics Letters A 380 (2016) 1735-1740.

[49] T.A. Ho, A. Striolo, Molecular dynamics simulation of the graphene-water interface: comparing water models, Molecular Simulation 40 (2014) 1190-1200.

[50] Kole M., Dey T. K.: Role of interfacial layer and clustering on the effective thermal conductivity of CuOgear oil nanofluids, Exp. Thermal and Fluid Sci. 35, 1490-1495 (2011).

[51] Z.H. Li, Y.J. Gong, M. Pu, D. Wu, Y.H. Sun, J. Wang, Y. Liu, B.Z. Don, Determination of interface layer thickness of a pseudo two-phase system by extension of the Debye equation, J. Phys. D: Appl. Phys. 34 (2001) 2085-2088.

[52] S.T. Wereley, R.M. Lueptow, Inertial particle motion in a Taylor Couette rotating filter, Physics of Fluids 11 (1999) 325-333.

[53] J.W. Wang, M.A. van der Hoef, J.A.M. Kuipers, The role of particle-particle interactions in bubbling gasfluidized beds of Geldart A particles: A discrete particle study, AIP Conference Proceedings (2010), 1207, 766.

[54] H. Bruus, Theoretical microfluidics, Lecture notes, Technical University of Denmark, Copenhague (2005).

[55] M.W. Reeks, On the constitutive relations for dispersed particles in nonuniform flows. I: Dispersion in a simple shear flow, Physics of Fluids A: Fluid Dynamics (1993) 5, 750.

[56] R. Pal, Evaluation of theoretical viscosity models for concentrated emulsions at low capillary numbers, Chemical Engineering Journal 81 (2001) 15-21.

[57] R. Pal, Modeling viscosity of concentrated nanoemulsions and nanosuspensions, Fluids 1 (2016) 11.

[58] M.A. Lauffer, Motion in viscous liquids, Journal of Chemical Education 58 (1981) 250-256.

[59] E. Walker, P.W.J. Glover, Permeability models of porous media: Characteristic length scales, scaling constants and time-dependent electrokinetic coupling, Geophysics 75 (2010) E235-E246.

[60] V.V. Guryev, V.I. Nikitsin, V.A. Kofanov, Determination of the hydraulic radius of the porous structure of ceramic materials (2016), Glass and Ceramics 73, 258-265.

[61] D. Leith, Drag on Nonspherical Objects (1987), Aerosol Science and Technology 6:153-161.

[62] D. Argyris, T. Ho, D.R. Cole, A. Striolo, Molecular dynamics studies of interfacial water at the alumina surface, The Journal of Physical Chemistry C 115 (2011) 2038-2046.

[63] N. Dolatabandi, R. Rahmani, H. Rahnejat, C.P. Garner, Thermal conductivity and molecular heat transport of nanofluids, RSC Adv. (2019) 9, 2516.

[64] F. Jabbari, A. Rajabpour, S. Saedodin, S. Wongwises, Effect of water/carbon interaction strength on interfacial thermal resistance and the surrounding molecular nanolayer of CNT and graphene flake, Journal of Molecular Liquids (2019) 282, 197-204.

[65] A. Akaishi, T. Yonemaru, J. Nakamura, Formation of water layers on graphene surfaces, ACS Omega 2 (2017) 2184-2190.

[66] T. Petit, H. Yuzawa, M. Nagasaka, R. Yamanoi, E. Osawa, N. Kosugi, E.F. Aziz, Probing interfacial water on nanodiamonds in colloidal dispersion, J. Phys. Chem. Lett. 6 (2015) 2909-2912.

[67] M.V. Korobov, N.V. Avramenko, A.G. Bogachev, N.N. Rozhkova, E. Osawa, Nanophase of water in nanodiamond gel, J. Phys. Chem. (2007) 111, 7330-7334.

[68] P. K. Namburu, D. P. Kulkarni, A. Dandekar and D. K. Das, Experimental investigation of viscosity and specific heat and Silicon Dioxide nanofluids, Micro \& Nano Letters, 2 (3) (2007) 67-71.

[69] J. Chevalier, O. Tillement and F. Ayela, Rheological properties of nanofluids flowing through microchannels (2008), Appl. Phys. Lett. 91, 233103. 
[70] E. V. Timofeeva, D. S. Smith, W. Yu, D. M. France, D. Singh and J. L. Routbo, Particle size and interfacial effects on thermo-physical and heat transfer characteristics of water-based $\alpha$-SiC nanofluids (2010), Nanotechnology 21, 215703.

[71] M.J. Pastoriza-Gallego, C. Casanova, J.L. Legido, M.M. Piñeiro, CuO in water nanofluid: influence of particle size and polydispersity on volumetric behavior and viscosity (2011), Fluid Phase Equilibria 300, 188-196.

[72] M. Baratpour, A. Karimipour, M. Afrand, S. Wongwises, Effects of temperature and concentration on the viscosity of nanofluids made of single-wall carbon nanotubes in ethylene glycol (2016), Int. Comm. Heat Mass Transfer 74, 108-113.

[73] M. N. Rashin, J. Hemalatha, Viscosity studies on novel copper oxide-coconut oil nanofluid (2013), Exp. Thermal Fluid Science 48, 67-72.

[74] A. Weiss, N. Dingenouts, M. Ballauff, H. Senff, W. Richtering, Comparison of the effective radius of sterically stabilized latex particles determined by small-angle x-ray scattering and by zero shear viscosity (1998), Langmuir14, 5083-5087.

[75] T. Jiang, C.F. Zukoski, Role of particle size and polymer length in rheology of colloid-polymer composites (2012), Macromolecules 45, 9791-9803.

[76] B.J. Anderson, C.F. Zukoski, Rheology and Microstructure of an unentangled polymer nanocomposite melt (2008), Macromolecules 41, 9326-9334.

[77] T.G. Mason, J. Bibette, D.A. Weitz, Yielding and flow of monodisperse emulsions (1996), J. Coll. Inter. Sci. $179,439-448$

[78] U. Bains, R. Pal, In-situ continuous monitoring of the viscosity of surfactant-stabilized and nanoparticlesstabilized pickering emulsions (2019), Appl. Sci. 9, 4044.

[79] M. Roullet, P.S. Clegg, W.J. Frith, Viscosity of protein-stabilized emulsions: contributions of components and development of a semipredictive model (2019), J. Rheology 63, 179.

[80] E. Harika, S. Jarny, P. Monnet, J. Bouyer, M. Fillon, Effect of water pollution on rheological properties of lubricating oil (2011), Appl. Rheol. 21, 12613.

[81] D.W. Green, M.Z. Southard, Perry's Chemical Engineer's Handbook, McGraw Hill Education, $9^{\text {th }}$ edition, (2018).

[82] C. Sivaram, A.S.N.D. Murthy, The effect of temperature variation on studies of coconut and palmoline oils (2010), Int. J. Chem. Sci. 8, 2259-2266.

[83] V. Bertola, An experimental study of bouncing Leidenfrost drops: comparison between Newtonian and viscoelastic liquids (2009), Int. J. Heat Mass Transfer 52, 1786-1793.

[84] T.Y. Wu, B.K. Chen, L.P. Hao, Y.C. Peng, I.W. Sun, Effect of Temperature on the Physico-Chemical Properties of a Room Temperature Ionic Liquid (1-Methyl-3-pentylimidazolium Hexafluorophosphate) with Polyethylene Glycol Oligomer (2011), International journal of molecular sciences 12, 2598-2617. 